\title{
Prognostic and Classification of Dynamic Degradation in a Mechanical System Using Variance Gamma Process
}

\author{
Marwa Belhaj Salem (D), Mitra Fouladirad * and Estelle Deloux \\ ICD-M2S, University of Technology of Troyes, 10000 Troyes, France; marwa.belhaj_salem@utt.fr (M.B.S.); \\ estelle.deloux@utt.fr (E.D.) \\ * Correspondence: mitra.fouladirad@utt.fr
}

Citation: Belhaj Salem, M.;

Fouladirad, M.; Deloux, E. Prognostic and Classification of the Dynamic

Degradation in a Mechanical System Using Variance Gamma Process. Mathematics 2021, 9, 254. https:// doi.org/10.3390/math9030254

Academic Editor: Vlad Stefan Barbu Received: 7 December 2020

Accepted: 21 January 2021

Published: 27 January 2021

Publisher's Note: MDPI stays neutral with regard to jurisdictional clai$\mathrm{ms}$ in published maps and institutional affiliations.

Copyright: $\odot 2021$ by the authors. Licensee MDPI, Basel, Switzerland. This article is an open access article distributed under the terms and conditions of the Creative Commons Attribution (CC BY) license (https:// creativecommons.org/licenses/by/ $4.0 /)$.

\begin{abstract}
Recently, maintaining a complex mechanical system at the appropriate times is considered a significant task for reliability engineers and researchers. Moreover, the development of advanced mechanical systems and the dynamics of the operating environments raises the complexity of a system's degradation behaviour. In this aspect, an efficient maintenance policy is of great importance, and a better modelling of the operating system's degradation is essential. In this study, the nonmonotonic degradation of a centrifugal pump system operating in the dynamic environment is considered and modelled using variance gamma stochastic process. The covariates are introduced to present the dynamic environmental effects and are modelled using a finite state Markov chain. The degradation of the system in the presence of covariates is modelled and prognostic results are analysed. Two machine learning algorithms k-nearest-neighbour (KNN) and neural network (NN) are applied to identify the various characteristics of degradation and the environmental conditions. A predefined degradation threshold is assigned and used to propose a prognostic result for each classification state. It was observed that this methodology shows promising prognostic results.
\end{abstract}

Keywords: variance gamma; stochastic models; degradation processes; covariates; classification; k-nearest-neighbour; neural network; artificial intelligence

\section{Introduction}

Recently advanced systems are becoming highly complex due to the integration of different subsystems and as a result, the maintenance of such high-priced systems is considered a challenging task for engineers. It has been established that the maintenance of such a system at appropriate times can assure the high reliability and safety of systems. As a consequence, reliability engineers and researchers introduced the degradation modelling to better predict the deteriorating system lifetime. Different stochastic models such as gamma process, Wiener process, etc. have been used to model the system degradation in the past [1-4]. The most popular process, the gamma process, was used when degradation monotonically accumulates over time, whereas the Wiener process is used when system degradation witnesses non-monotonic behaviour. In this paper, the degradation of a centrifugal pump operating in a dynamic environment is considered. As the centrifugal pump exhibits non-monotonic behaviour and complex degradation, the scope of new sophisticated non-monotonic stochastic models rises. As an alternative to the Wiener process, we propose the variance gamma process to model system degradation. Considering the impact of dynamic environmental conditions, the classification of degradation data is carried out using two machine learning algorithms and a lifetime prediction.

The variance gamma (VG) process was first introduced in 1990 by Madan and Seneta [5] as a new stochastic process for financial analysis. Continuous time specification, long tailedness, finite moments of all orders, good empirical fit, etc. are the main advantages of this process. They have several characteristics such as pure jump, approximable by a compound Poisson process with a high jump frequency, and low jump magnitudes. 
The main purpose of introducing the VG process was to substitute a common diffusion process such as the Black \& Scholes (B \& S) model for a non-monotonous and the positive trend modelling. VG can offer a better fit compared to (B \& S) by providing low variances and high kurtosis [6]. VG process consists of four parameters that are developed as a generalised Brownian motion. It can be achieved by considering a Brownian motion with drift at a random time given by a gamma process. The presenting parameters allow control over the kurtosis and skewness of the increments distribution. The statistical density is observed to be symmetric with some kurtosis [7]. Several studies were performed in the past using the VG process [8-15], but the practicability of VG in modelling system degradation has never been explored in the past.

The integration of the covariates in lifetime modelling was introduced in 1972 by Cox using the regression analysis proportional hazard model (PHM) [16]. This model has been chosen for the evaluation of reliability in the presence of covariates. Since then, several studies have focussed on the reliability and maintenance of the system considering covariates. Deloux et al. [17] studied the maintenance optimisation of a deteriorating system and a statistical process control was used to track the potential drift in the stress covariate presenting the shock. Zhao et al. [18] proposed a maintenance model for single-unit systems whose degradation is influenced by the number of inspections. They developed an efficient parameter estimation procedure considering the environmental covariates. Barabadi et al. [19] investigated the efficiency of the reliability models with covariates using the example of the spare part predictions. They investigated the effect of the geological characteristics of the rock, the operating history of the machine, the material, operator, etc., on the degradation of the system in order to provide a better spare parts forecasting. Okaro and Tao [20] proposed the Weibull-Corrosion covariate model to assess the reliability of a subsea compression system working under pressure taking into consideration the effect of corrosion on the degradation. Moniri-Morad et al. [21] evaluated the reliability of mining equipment subject to the dominant explanatory variables impact and reduced the equipment failure intensity. Slimacek and Lindqvist [22] analysed the reliability of wind turbines by proposing the Poisson process as a degradation model taking into account covariates such as type of turbine, size of the turbine, harshness of the environment, installation date, and seasonal effects and compared it to the model not taking into account the unobservable cited covariates. Duan and Wang [23] evaluate the reliability problem for products using the exponential-dispersion (ED) process incorporating the random effects and covariates like the environmental factors. Barabadi et al. [24] proposed the efficient Cox regression model and its extension to model the presence of the operational environment covariates. Guo and Love [25] investigated the interactions between an industrial system and its environment. The proposed model takes into account the system degradation and the covariates such as the industrial environment. Zhu et al. [26] studied the degradation of the system that consist of wear damages and random accumulative shock damage caused by environmental conditions. The system degradation is modelled and the dynamic environment effects presented as covariates are modelled using the Cox proportional hazards model. Zhang et al. [27] studied the reliability and the prognostic of a k out of $\mathrm{N}$ system in which the failure of each component is subjected to the presence of covariates like the operating environment conditions. The calculation of the reliability and the expected remaining useful lifetime of the system are presented. Lawless and Crowder [28] proposed a gamma process with the presence of covariable like the random effect in order to fit to the crack growth data. Laucelli et al. [29] investigated the degradation of the pipes in the presence of covariates like the climate data and proposed the Evolutionary Polynomial Regression as a model. Park et al. [30] proposed PHMs to model the times between consecutive pipe breaks. The time dependent effects of the environment effects known as covariates on the failure hazard in the models were also included. Balekelayi et al. [31] modelled the degradation of sewer pipes using a Bayesian geoadditive regression model incorporating physical and environmental covariates. The model was applied to inspect pipes which allow the urban water utility managers in their prioritisation of inspection, 
maintenance, and replacement. Similarly, several works were performed in different degradation system modelling [32-44].

Hong et al. [45] studied the outdoor weathering degradation data in the presence of dynamic covariates information (i.e., changing UV spectrum and intensity, temperature, and humidity). The general path model is used with individual random effects as a degradation path model and the covariate process is described using a vector time series model. $\mathrm{Si}$ et al. [46] proposed a novel accelerated degradation testing (ADT) model by integrating the covariates effect such as the long-term degradation memory effect. Simulation studies were implemented to illustrate the efficiency of the developed methods. Aalen et al. [47] investigated that the estimation of the Markov chain transition probabilities depend on covariates. Islam and Chowdhury [48] studied a covariate dependent Markov model and provided a comprehensive explication for the higher order. Sirima and Pokorny [49] proposed a two-state hidden Markov model (HMM) to describe the effect covariate such as machine part error correction and also the time spent on the error correction of defective industrial machine parts. Other researchers also studied the modelling of the covariates using the Markov chain [50,51].

The real retrieved data from large-scale systems operating in a dynamic environment provide different information. The presence of the covariates can affect the level of degradation and it is essential to sort degradation information. In this study, two well known artificial intelligence (AI) algorithms for classification such as k-nearest neighbours (KNN) and neural network $(\mathrm{NN})$ algorithms are proposed in order to classify data. The KNN algorithm was initially proposed by Thomas Cover [52] as a supervised machine learning (ML) algorithm capable of insuring both classification as well as regression predictive problems. It was mainly used to predict the problems in the industry and to recognise the different patterns. Di et al. [53] used the KNN algorithm to classify the incoming maintenance requests. They proposed an automatic system able to classify the incoming maintenance requests and, without any human intervention, organise them with the lowest error and biggest respect to the routing policy. Yao et al. [54] focused on applying a KNN query processing in tracking objects using sensor networks. They analysed the optimal maintenance of the monitoring area and developed an adaptive algorithm to actively decide when to reduce the monitoring area. Wang et al. [55] suggested the KNN algorithm to realise the predictive maintenance of rolling bearings in the industry. Lee et al. [56] presented an innovative methodology to predict the breakdown of the machine. A pattern discrimination model (PDM) based on a neural network was developed to investigate the behaviour of the machine quantitatively. Huang et al. [57] introduced statistical analysis to determine the important elements that influence the degradation and create a model in order to estimate the future condition of the bridges. Several important elements were identified and the NN model was developed to predict degradation. Quah et al. [58] applied neural network to estimate software quality including both the reliability and maintainability of software estimation using an object-oriented metric. They used two neural network models: Ward neural network and general regression neural network (GRNN). It was concluded that the GRNN network model is better than the ward network model in prediction.

The aim of this study is to propose a non-monotonic stochastic process variance gamma to model the degradation of a mechanical system operating in the dynamic environment. The covariates will be introduced to present the dynamic environmental effects, and they will be modelled using a time continuous finite state Markov chain. The degradation of the system in the presence of covariates will be modelled to propose an efficient prognostic. Two machine learning algorithms, $\mathrm{KNN}$ and $\mathrm{NN}$, will be applied to identify the various characteristics of the degradation. A predefined degradation threshold will also be assigned and used to propose a good prognostic for each classification state. The remainder of this paper is structured as follows. A presentation of the VG process will be detailed in Section 2. The centrifugal pump system and the data retrieved is presented in Section 3. The centrifugal pump operates in a dynamic environment that can be presented using 
covariates. The modelling of the operating system taking in account of the covariates will be detailed in Section 4. To propose an efficient prognostic, two classification methods will be proposed to classify the system's degradation in Section 5. Conclusion of the paper is presented in Section 6.

\section{Variance Gamma Process}

VG process was introduced in 1990 by Madan and Seneta [5]. This process can be attained by evaluating the Brownian motion with drift at a random time given by a gamma process. Let $b(t ; \theta, \sigma)$ be a process that is considered as a Brownian motion with drift $\theta$ and volatility $\sigma$ and $\mathrm{W}(\mathrm{t})$ as a standard Brownian motion. It can be expressed as follows:

$$
b(t ; \theta, \sigma)=\theta t+\sigma W(t), \quad \sigma>0, \quad t>0
$$

Consider a gamma process $\gamma(t ; \mu, v)$ where $\mu$ is the mean rate and $v$ is the variance rate of the process. The obtained gamma density function $f_{h}(g)$ of increment $g(h)=$ $\gamma(t+h ; \mu, v)-\gamma(t ; \mu, v)$ with mean $\mu h$ and variance $v h$ can be obtained by considering gamma function $\Gamma(x)$ as:

$$
f_{h}(X)=\frac{1}{\sigma \sqrt{2 \pi \gamma(t+h)-\gamma(t)}} \exp \left(-\frac{(X-\theta \gamma(t+h)-\gamma(t))^{2}}{2 \sigma^{2} \gamma(t+h)-\gamma(t)}\right) \frac{\gamma(t+h)-\gamma(t)^{\frac{t}{v}-1} \exp \left(-\frac{\gamma(t+h)-\gamma(t)}{v}\right)}{v^{\frac{t}{v}} \Gamma\left(\frac{t}{v}\right)}
$$

The VG process is defined as an evaluation of the time of a Brownian motion by a gamma process, written as:

$$
X(t ; \sigma, v, \theta)=b(\gamma(t ; \mu, v), \theta, \sigma)
$$

Moreover, the variance gamma process can be expressed as the difference of two gamma processes due to its definition as a process of finite variation. The two gamma processes illustrate two competitive phenomena where the first gamma can express the increase in the process and the second expresses a decreasing in the process. For that, the VG process can be presented as a difference of two independent gamma processes and can be written as follows:

$$
X(t ; \sigma, v, \mu)=\gamma_{p}\left(t ; \vartheta_{p}, v_{p}\right)-\gamma_{n}\left(t ; \vartheta_{n}, v_{n}\right)
$$

The link between the parameters of the VG process and those of the difference between the gamma process parameters are represented as:

$$
\begin{array}{ll}
\vartheta_{p}=\frac{1}{2} \sqrt{\vartheta^{2}+\frac{2 \sigma^{2}}{v}+\frac{\vartheta}{2}} \quad v_{p}=\left(\frac{1}{2} \sqrt{\vartheta^{2}+\frac{2 \sigma^{2}}{v}}+\frac{\vartheta}{2}\right)^{2} v \\
\vartheta_{n}=\frac{1}{2} \sqrt{\vartheta^{2}+\frac{2 \sigma^{2}}{v}}-\frac{\vartheta}{2} & v_{n}=\left(\frac{1}{2} \sqrt{\vartheta^{2}+\frac{2 \sigma^{2}}{v}}-\frac{\vartheta}{2}\right)^{2} v
\end{array}
$$

Figure 1 presents a sample path of the variance gamma process. Non-monotonic behaviour of the VG process with high pure jumps can clearly be observed from the path. 


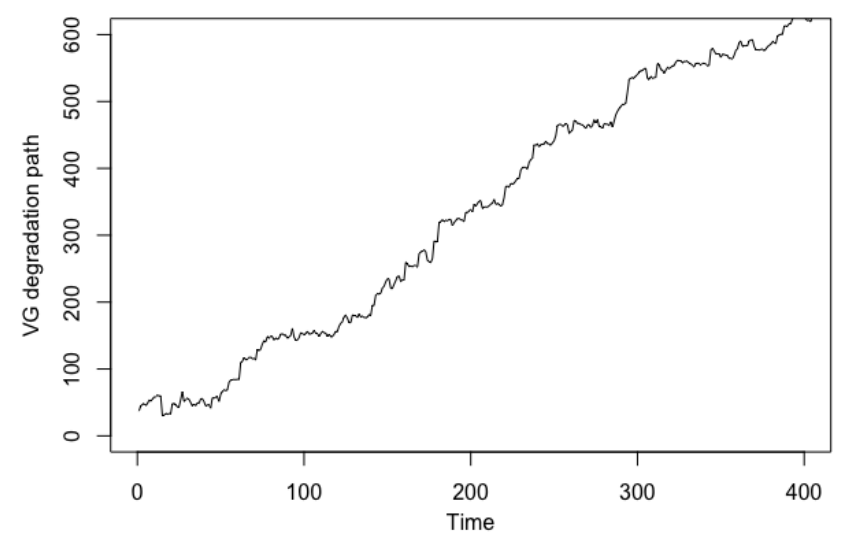

Figure 1. Variance gamma sample path.

The density function of the VG process at time $t$ given by $f_{X(t)-X(s)}(X)$ can be expressed as follow:

$$
f_{X(t)-X(s)}(X)=\frac{1}{\sigma \sqrt{2 \pi g}} \exp \left(-\frac{(X-\theta g)^{2}}{2 \sigma^{2} g}\right) \frac{g^{\frac{t-s}{v}-1} \exp \left(-\frac{g}{v}\right)}{v^{\frac{t-s}{v}} \Gamma\left(\frac{t}{v}\right)} .
$$

The flexibility of the VG process due to its two extra parameters permits the model to fit different non-linear degradation problems. Moreover, VG can be represented as an evaluation of a Brownian motion time by a gamma process and a difference of two gamma processes, which allow it to model different degradation phenomena.

\section{Environmental Conditions and Failure Time}

\subsection{Environmental Conditions: Covariates}

Recent studies show that mechanical systems are subject to numerous environmental conditions such as temperature, vibrations, humidity, shock, etc. Conventional models do not take into account these factors and the effects caused by these external factors that can influence the system's lifetime. These external factors will be defined here as covariates. The stressful conditions' effects tend to decrease system reliability leading to unexpected system behaviour and an increase in operating costs. The sensitivity studies indicate that the covariates impacts the system degradation modelled using the variance gamma process through its two parameters, $\sigma$ and $\theta$.

In this study, the environmental conditions are presented by a $Z=\left(Z_{t}\right)_{t \geq 0}$ homogeneous Markov chain with finite state space $\{1,2, \ldots, \mathrm{m}\}$ and $m \in \mathbb{N}^{*}$. The impact of the covariates is detectable only by inspections. The environment impacts the degradation of the system by affecting the values of $\sigma$ and $\theta$. For each state, presenting a type of degradation is assigned based on the values of $\sigma$ and $\theta$. Assuming the homogeneity of the covariates process $Z$, the transition probabilities do not depend on $n$. Let $p_{i j}=\mathbb{P}\left(Z_{n+1}=j \mid Z_{n}=i\right) \quad \forall n \in \mathbb{N}^{*}$ be the proposed transition probabilities of $Z$, the transition matrix of the process $Z$ will be denoted by $P=\left(p_{i j}\right)$. The first state of the chain is $Z_{0}=1$, and given as $\pi^{n}=\left(\pi_{1}^{n}, \pi_{2}^{n}, \pi_{3}^{n}\right)$ where $\pi_{i}^{n}=\mathbb{P}\left(Z_{n}=i\right),(i=1,2,3)$ is the distribution of $Z_{n}$. $\left(\pi_{1}^{n}, \pi_{2}^{n}, \pi_{3}^{n}\right)=\mathbb{P}\left(Z_{n}=1 \mid Z_{0}=1\right), \mathbb{P}\left(Z_{n}=2 \mid Z_{0}=1\right), \mathbb{P}\left(Z_{n}=\right.$ $\left.3 \mid Z_{0}=1\right)=(1,0,0) P^{n}$, and $\lim _{n \rightarrow \infty} \pi_{i}^{n}=\pi_{i}$ is the stationary distribution of the Markov chain.

Let $\Theta$ presents the vector of the parameters to estimate $\Theta=\left(\mu, v, \theta_{1}, \theta_{2}, \ldots, \theta_{m}, \sigma_{1}, \sigma_{2}\right.$ $\left.\ldots \sigma_{m}\right)$, considering a random sample $x=\left(x_{1}, x_{2}, \ldots x_{k}\right)$, where $\theta_{i}$ and $\sigma_{i}$ presents the parameters of the VG process for each state $i$. 
The maximum likelihood with a m-state Markov chain can be written as:

$$
\begin{aligned}
L(x, \Theta)= & \prod_{i=1}^{n} \sum_{j=1}^{n} f_{\Theta}\left(x_{i}\right)=\frac{2}{(2 \Pi)^{\frac{n}{2}}} \prod_{i=1}^{n} \sum_{j=1}^{n} \frac{\pi_{i}}{\sigma_{j} v^{\left(\frac{1}{v}\right)} \Gamma\left(\frac{1}{v}\right)}\left(\frac{1}{\frac{2 \sigma_{j}^{2}}{v}+\theta_{j}^{2}}\right)^{\frac{1}{v}-\frac{1}{2}} \times \\
& \exp \left(\frac{\theta_{j}\left(x_{i}-\mu\right)}{\sigma_{j}^{2}}\right)\left|x_{i}-\mu\right|^{\frac{1}{v}-\frac{1}{2}} \times \kappa_{\frac{1}{v}-\frac{1}{2}}\left(\frac{\left|x_{i}-\mu\right| \sqrt{2 \sigma_{j}^{2} v+\theta_{j}^{2}}}{\sigma_{j}^{2}}\right) .
\end{aligned}
$$

\subsection{Failure Time}

It is important to evaluate the evolution of degradation and thereby predict the system's failure. In this context, the failure time that is the first time the degradation paths exceed the predefined threshold is set at $L$. The conventional definition of the first hitting time cannot be applied in the case of a VG process because of the non continuity of the subordinator gamma. Hurd $[59,60]$ introduced the first hitting time of the second kind which present the first time when the gamma process exceeds the failure time of the Brownian motion (b). Considering $T_{F}^{*}$ to be the first failure time of the Brownian motion $B$ which can be written as:

$$
T_{F}^{*}=\inf \left\{t: x_{0}+\theta t+\sigma W(t) \geq 0, \quad t \geq 0\right\}
$$

The failure time of the VG process $X_{t}=B_{G_{t}}$ is introduced as:

$$
t_{L}^{*}=\inf \left\{t: G_{t} \geq T_{F}^{*}\right\}
$$

The new concept provides some advantages like the efficiency of calculation compared to the normal failure time calculation. Another alternative approximation is the cumulative distribution function (CDF) of the failure time (FT) obtained by the following equation:

$$
\mathbb{P}\left(t_{L}^{*}<t\right)=2 \mathbb{P}(X(t)>L)=2\left(1-F_{X(t)}(L)\right)
$$

where the $F_{X(t)}(L)=\int_{-\infty}^{d} f_{X(t)}(x) d x$ and the $f_{X(t)}$ is given by Equation (6) and $L$ is the predefined threshold.

Another analytical approximation of the FT and the CDF of the FT were presented by $\mathrm{Li}$ [61]. It can be obtained by using the following equations:

$$
\begin{gathered}
\frac{1}{2} \int_{0}^{1}\left[1+\operatorname{erf}\left(-\frac{\ln \left(\frac{1}{d}\right)}{\sigma \sqrt{-2 v \ln (y)}}-\frac{\mu}{\sigma \sqrt{2}} \sqrt{-v \ln (y)}\right)\right] \cdot \frac{(-\ln y)^{\frac{T}{v}-1}}{\Gamma\left(\frac{T}{v}\right)} d y \\
\frac{1}{2} \exp \left(\frac{-2 \mu \ln \left(\frac{1}{d}\right)}{\sigma^{2}}\right) \int_{0}^{1}\left[1+\operatorname{erf}\left(-\frac{\ln \left(\frac{1}{d}\right)}{\sigma \sqrt{-2 v \ln (y)}}+\frac{\mu}{\sigma \sqrt{2}} \sqrt{-v \ln (y)}\right)\right] \cdot \frac{(-\ln y)^{\frac{T}{v}-1}}{\Gamma\left(\frac{T}{v}\right)} d y
\end{gathered}
$$

It is important to present the analytical definition of the remaining useful life (RUL) in order to predict the time left before the degradation measure crosses the $L$ failure threshold and the system loses its operating capability. The expression of the RUL is presented as follows:

$$
R U L(t)=\left\{h>0, \quad X_{t+h}>L \mid X_{t}<L\right\}
$$

\section{Centrifugal Pump System and Degradation Data}

\subsection{Presentation of Degradation Data}

The degradation of the centrifugal pump system is considered in this study. The centrifugal pump system is used in several large and small-scale industries. The small defect in this pump can cause a huge economic loss and catastrophic damages to the entire system. The main cause of the degradation of a centrifugal pump system is caused by the damage of the seals. The degradation of a system is monitored over different periods and based 
on one chosen health indicator, such as the leakage rate expressed with a unit measurement as (L/h: litre per hour). The real retrieved data presenting the health indicator of centrifugal pumps over a determined period are presented and will be applied to confirm the usefulness of the VG process as a degradation model. The data are obtained over nine different time span and contain information from four different sensors and will be used in this study to calibrate the degradation model. Figure 2 presents the leakage rate of the four systems over a time span. It shows the behaviour of the degradation since the leakage rate (L/h: litre per hour) presents the degradation indicator over the time (h: hour). Based on the historical information of the system, the degradation of the pump is mainly caused by the independent failure of its seals. In order to detect seal failure earlier, potential degradation modes are detected by the operational monitoring of the seal leak rates. The histogram of the four systems degradation paths real increments are presented in Figure 3.

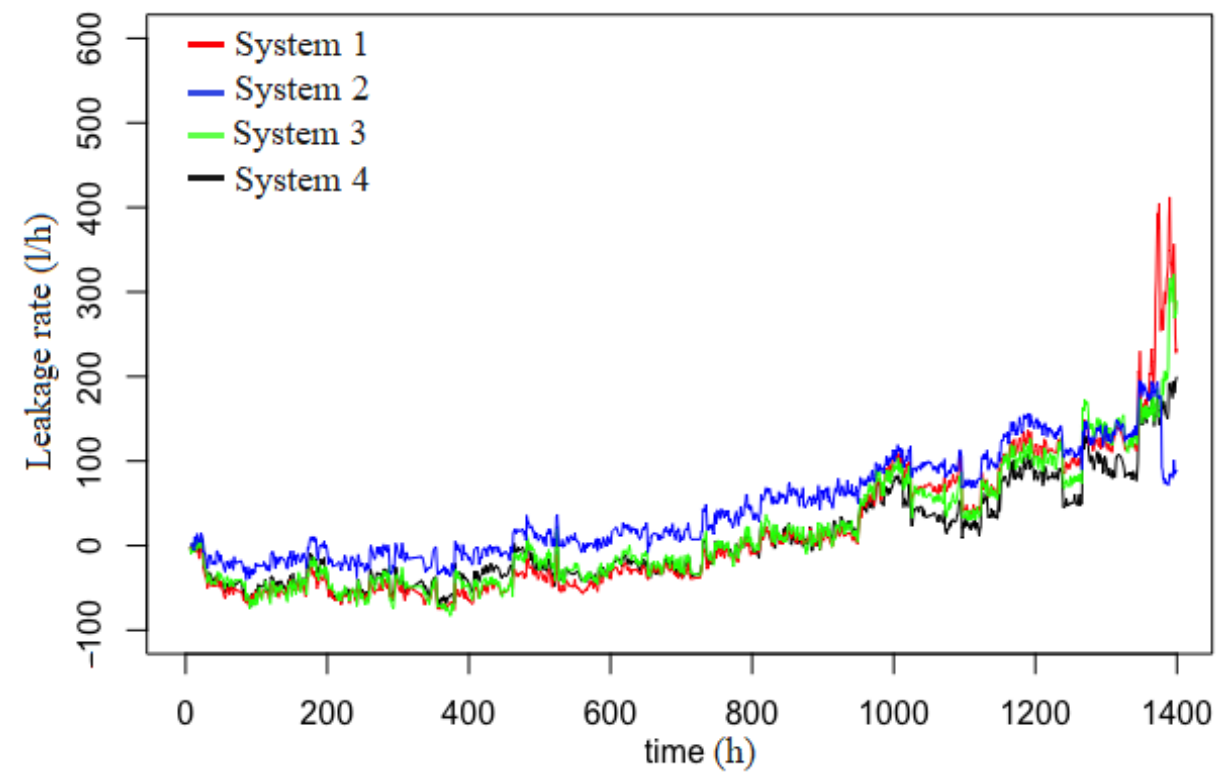

Figure 2. Leakage rate $(\mathrm{L} / \mathrm{h})$ of the four systems over a time $(\mathrm{h})$.

The main focus is only on the data describing the leak rate of seal pumps which constitute the degradation indicator. As soon as the seal's leak rate of one of the pumps exceeds a limit set at around $1400 \mathrm{~L} / \mathrm{h}$, the system is stopped (more or less quickly) and the seal of the pump under consideration is replaced. This event technically represents the failure of the pump seal. An alarm threshold has been set at a leak rate of approximately $1100 \mathrm{~L} / \mathrm{h}$, in order to alert the operator early enough of a possible degradation of one of the seals and to be able to modify the operating conditions of the unit or carry out a maintenance operation to avoid breakdowns. The task after analyzing the data is to adapt an appropriate model that best describes the degradation phenomena, give a precise prognostic, and thereby propose an efficient maintenance policy. Finally, it is essential to verify the data in order to obtain the behaviour of the system. On the basis of Figure 3, it is shown that the different histograms of the four systems increments are symmetrical in all cases. The peaks represent the most common values and it can be observed that data dissemination varies but not significantly. 


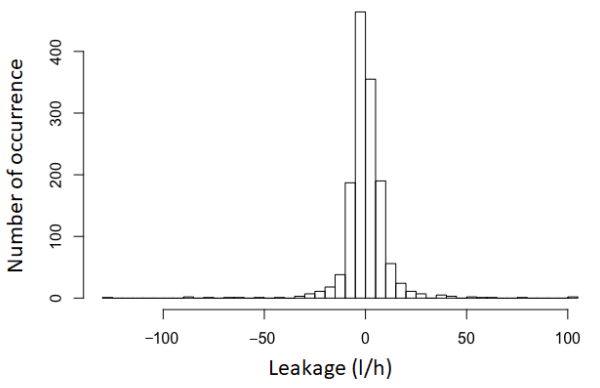

(a)

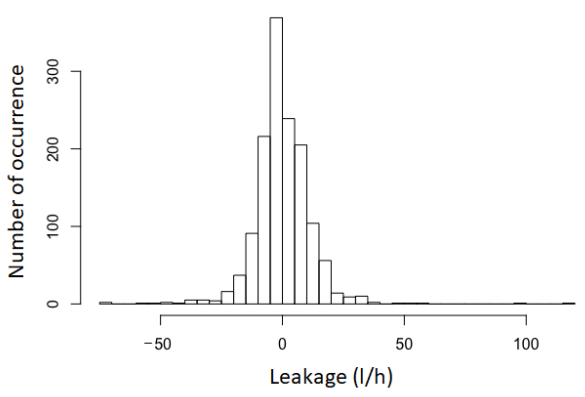

(c)

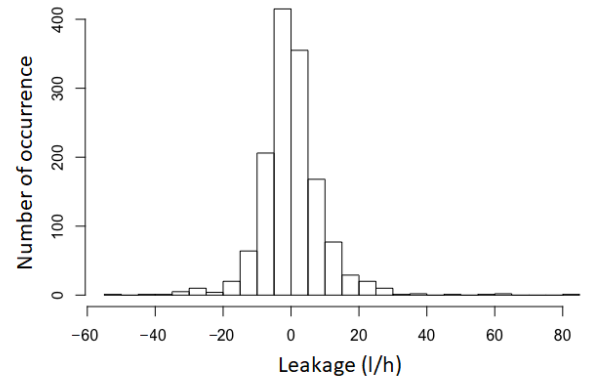

(b)

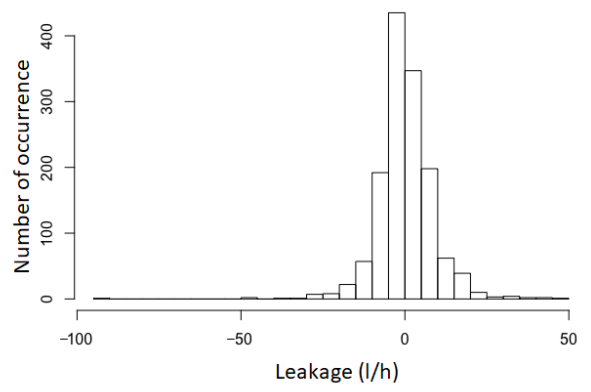

(d)

Figure 3. Histogram of the four systems degradation data (a) System 1, (b) System 2, (c) System 3, (d) System 4.

\subsection{Degradation Model with Covariates}

As described earlier, the pump operates in a dynamic environment which implies the necessity of integrating the impact of the environment on system degradation. The environmental impact is introduced using covariates and will be modelled by a finite state Markov chain. The environmental condition such as temperature, pressure, and humidity induces changes in the values of $\sigma$ and $\theta$. Based on the obtained degradation data, the speed of the degradation can be characterised into three types: Slow, moderate, and fast degradation. The different covariates impacts directly the values of the two parameters $\sigma$ and $\theta$. A sensitivity study proves that the two parameters control the system's degradation. For that, three states of the Markov chain are utilised to define three types of degradation (slow, moderate, and fast) presenting the influence of the covariates on the system's degradation.

In this study, each environmental condition of the system is presented by a state of a Markov chain. For $Z(t)=1$, the degradation is considered as a slow degradation, $Z(t)=2$ and $Z(t)=3$ presents the moderate and fast degradation respectively.

Let $p_{i j}=\mathbb{P}\left(Z_{n+1}=j \mid Z_{n}=i\right) \quad \forall n \in \mathbb{N}^{*}$ be the transition probabilities of the homogeneous process $Z$.

For a three states Markov chain, the transition matrix $P=\left(p_{i j}\right)$ can be presented as follows with $\lambda \in[0,1]$.

$$
P=\left[\begin{array}{ccc}
\lambda & 1-\lambda & 0 \\
\frac{\lambda}{2} & 1-\lambda & \frac{\lambda}{2} \\
1-\frac{\lambda}{2} & 0 & \frac{\lambda}{2}
\end{array}\right]
$$

For the real data, the parameters of the VG will be estimated and based on the values of the $\sigma$ and $\theta$ parameters, the three classes will be defined. For a small value of $\sigma$ and $\theta$ the degradation is considered to be slow, average and big values of $\sigma$ and $\theta$ induces moderate and fast degradation respectively. Figure 4 presents the difference between the three degradation states obtained after estimating the parameters of real data, based also on the values of both $\sigma$ and $\theta$ parameters. 


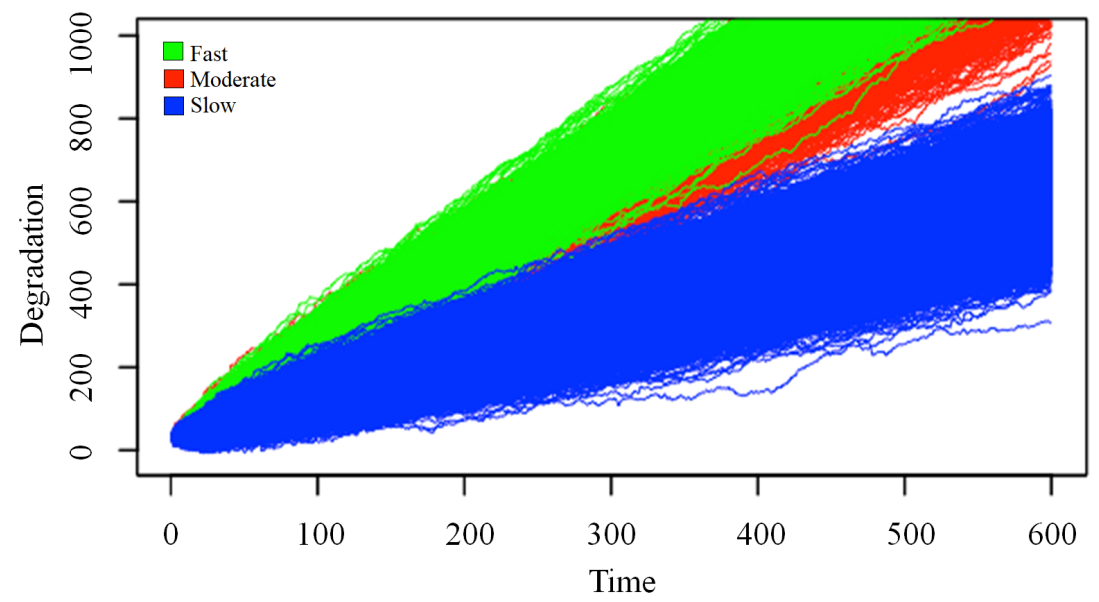

Figure 4. Degradation paths for different degradation state.

Figure 5 resumes the evolution of the degradation paths in the presence of the covariates for real data. It is evident that in the case of slow degradation, the degradation paths reaches the value of $100 \mathrm{~L} / \mathrm{h}$, moderate degradation paths reaches $600 \mathrm{~L} / \mathrm{h}$, and fast degradation paths reaches quickly the level of $1000 \mathrm{~L} / \mathrm{h}$ of leakage at the same period of time $t=150 \mathrm{~h}$. It also presents the fluctuation of the three covariates to express their impact on degradation speed.
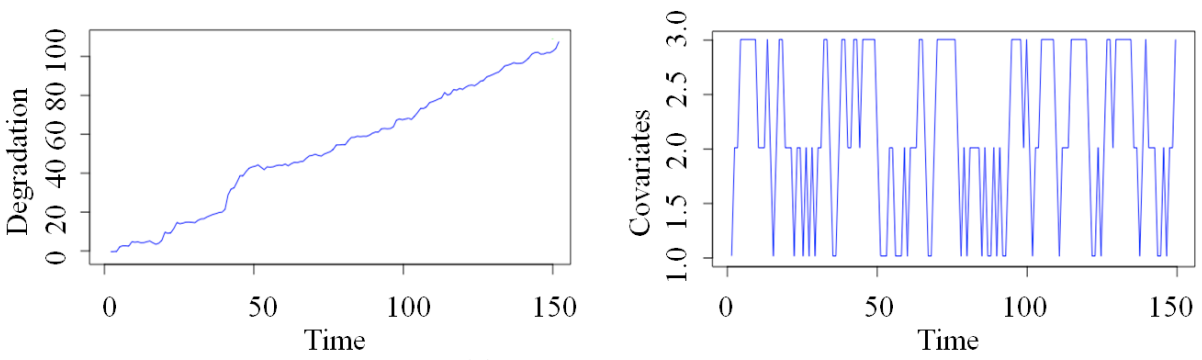

(a) Slow Degradation
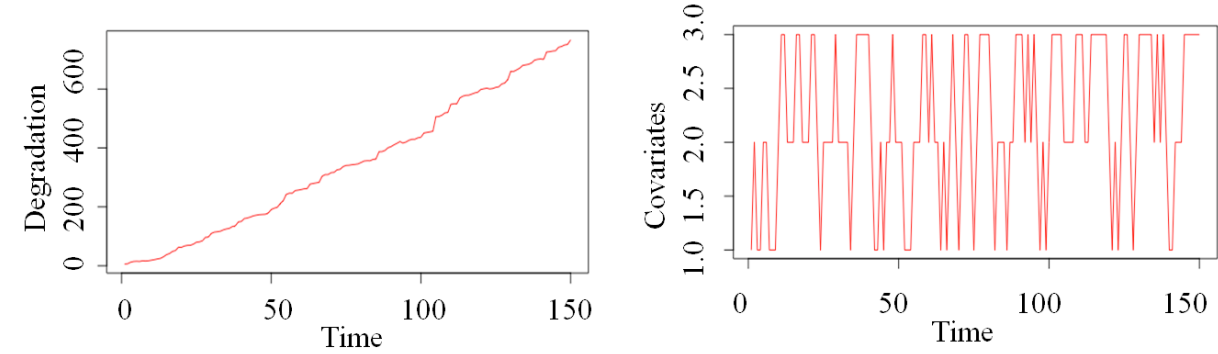

(b) Moderate Degradation
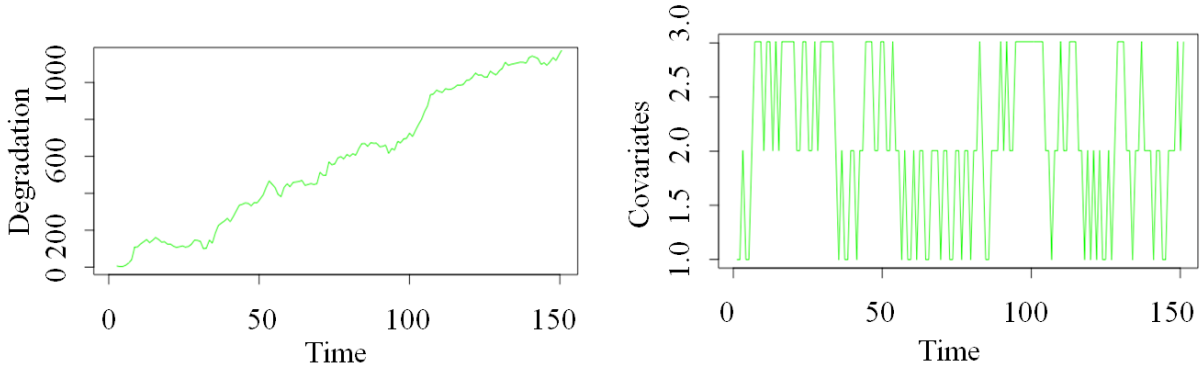

(c) Fast Degradation

Figure 5. Degradation paths and covariates for different degradation states based on real data. 
As the degradation behaviour can be grouped and modelled by three speeds: Slow, moderate, and fast. The covariates will be presented by a 3 state of a Markov chain and for each state the degradation paths follow the VG distribution given in Equation (6). Various degradation behaviours are presented using different VG parameters. Once the transition probabilities $p_{i 1}, p_{i 2}$, and $p_{i 3}$ are calculated and using the transition matrix of the 3 state Markov chain, the value of $\lambda$ can be obtained. Here, the $\lambda$ is calculated as 0.3.

Even though the simplification is applied, the estimation of the system's parameters with covariates is a difficult task to accomplish. The complexity of the calculation is due to the presence of the Bessel function of the third kind in the three expressions of the VG presenting the different covariates. The Bessel functions made the computation heavier and more sophisticated. To overcome these limitations, the supervised KNN classification method is proposed. The covariates will be modelled in three clusters describing the type of degradation: Slow, moderate, and fast. Once the classification task is presented, the rest of the prognostic work will be investigated for the different degradation data of the three clusters.

\section{Classification Methods}

In this section, the classification methods such as the $\mathrm{KNN}, \mathrm{NN}$ algorithms are proposed to overcome some limitation of calculation encountered before. The KNN algorithm and NN algorithm will be used to classify the type of degradation on both simulated and real data. The decision of the classification is based on the values of $\sigma$ and $\theta$ studied above. For $\theta<1.75$ and $\sigma<2.5$, the system degradation is considered to be slow. For $2<\theta<4$, and $2.5<\sigma<5$, the system is witnessing a moderate degradation. When $\sigma>5$ and $\theta>4$, the degradation is considered to be a fast degradation. For both methods, the total number of used data is equal to 300 observations that will be divided into two data sets: Training data and test data. From the data, 208 degradation data will be used to ensure the training phase, whereas 92 will ensure the test phase of both KNN and NN algorithms.

\subsection{Classification Methods for Simulated Data}

\subsubsection{K-Nearest-Neighbour Algorithms}

The KNN algorithm is used in this study to classify the type of degradation in order to propose an efficient prognostic. Firstly, the training data is generated to model different degradation types and will be used to classify the degradation of the pump on three clusters (each containing 100 candidates). The first class describes a slow degradation " $\mathrm{S}$ ", the second class describes a moderate degradation " $\mathrm{M}$ ", and the third class describes a fast degradation " $\mathrm{F}$ ". Initially, the KNN algorithm is applied on simulated data to test its potential and later it is applied on real data.

Firstly, the simulated data is used to evaluate the efficiency of the KNN algorithm. The VG process is used with different sets of parameter to present a slow, moderate, and fast degradation class. A total of 300 simulated data are used and will be presented by seven numeric features such as VGmod, the VGmean, the VGskew, the VGKurtosis, the VarVG, the minVG, and the maxVG as well as a target as a type of degradation 'Deg type'. The classification is based on the calculation of the Euclidean distance between the new VG degradation paths and its KNN. The calculation is based on the values of the features of the new VG degradation path. After building the data set, it is time to choose the optimal number of neighbors $K$. The number of neighbors $(K)$ in $K N N$ is a hyperparameter needed to be chosen at a model building time. The number $K$ can be seen as a controlling feature in the prediction model. According to researchers, there is no optimal number of neighbours, which suits all kinds of data sets. The KNN method was applied on the simulated data and for different values of $K$.

As mentioned before, the data set used in this article consists of 300 observations with eight features: Seven numeric features and one categorical feature presenting the degradation type. During the training and test tasks the first feature VG process can be removed as it does not provide useful information. The feature 'degradation type' is 
considered as the target feature. This variable will determine the results of the diagnosis based on the seven numeric features. The training and prediction data are investigated and presented to estimate how accurately the algorithm can predict the type of degradation. The computation of the accuracy is ensured by comparing actual test set values and predicted values. The prediction efficiency results of the KNN classification method for different values of $\mathrm{K}$ are presented in Table 1.

Table 1. K-nearest-neighbour (KNN) prediction accuracy for simulated data.

\begin{tabular}{lcccccc}
\hline $\boldsymbol{K}$ & $\mathbf{1}$ & $\mathbf{2}$ & $\mathbf{4}$ & $\mathbf{6}$ & $\mathbf{8}$ & $\mathbf{1 0}$ \\
\hline Accuracy & 0.6833 & 0.7945 & 0.8256 & 0.9013 & 0.8513 & 0.8386 \\
Classification rate & $68.33 \%$ & $79.45 \%$ & $82.56 \%$ & $90.13 \%$ & $85.13 \%$ & $83.86 \%$ \\
\hline
\end{tabular}

From the results, it is observed that when the value of $K$ is 1 , the predictions became less stable. Inversely, when the value of $K$ is increased, the predictions become more stable due to majority voting. The prediction accuracy increases from $79.45 \%$ to $82.56 \%$ and to $90.56 \%$ when the value of $K$ passes from $K=2$ to $K=4$ and then to $K=6$ and then start to decrease. For $K=8$ and $K=10$, the prediction accuracy decreases from $85.13 \%$ to $83.86 \%$. The obtained results show that the best algorithm accuracy was obtained for $K=6$ and is equal to $90.13 \%$. The results obtained for $K=8$ and $K=10$ are quite satisfying and equal to $85.13 \%$ and $83.86 \%$ respectively, but will not be considered. The KNN algorithm is implemented to ensure the degradation classification such that a prognostic analysis can be provided later. The decrease of the prediction accuracy results approves the choice of $K=6$ since any error of prediction can cause a huge error in the system's lifetime prognostic. At $t=0$, for a chosen degradation trajectory $\left(T_{\text {deg }}\right)$ modelled using the VG process, the parameters will be estimated and used to generate $\mathrm{N}=10,000$ different degradation trajectories. A predefined alarm threshold is settled and will be used to define the RUL distribution. The first time where these degradation trajectories cross the alarm threshold will be captured and used to define the RUL distribution. The aim is to assess the evolution of the RUL distribution. At a different inspection time $t=t_{\text {insp }}$, new degradation trajectories are generated to obtain a new RUL distribution. When the RUL distribution is obtained, the real crossing time of the $\left(T_{\text {deg }}\right)$ trajectory is located and compared to the RUL distribution, which can help us to provide an idea on the quality of the prognostic. The same work will be reproduced for different $\left(T_{\text {deg }}\right)$ trajectories presenting the three types of degradation: Slow, moderate, and fast. Figure 6 compares the position of the real crossing time $R_{c t}$ to the obtained histograms of RUL for the different degradation paths.

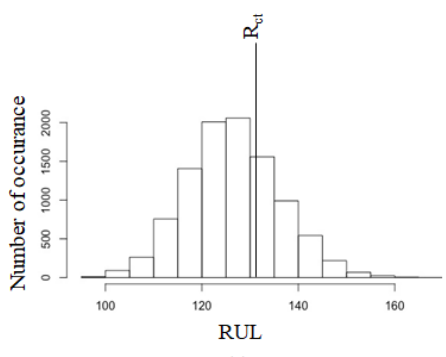

(a)

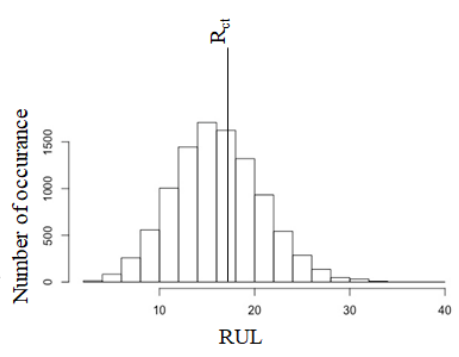

(b)

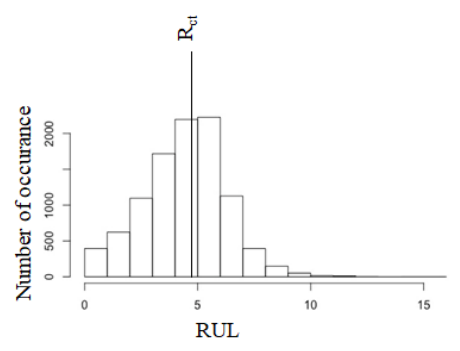

(c)

Figure 6. Position of the real crossing time $R_{c t}$ compared to histograms of RUL for simulated data with KNN algorithm at $t=0$ : (a) slow, (b) moderate, and (c) fast.

The first histogram presents the histogram of slow degradation where the degradation paths reached the failure threshold after $100 \mathrm{~h}$ of operation. The second histogram presents the degradation time. The system began its degradation, starting from $10 \mathrm{~h}$ of operating. For the last histogram, in the presence of a fast degradation the failure of the system occurs even before operating for $10 \mathrm{~h}$. 
The real crossing time $R_{c t}$ of the degradation trajectory $\left(T_{d e g}\right)$ for different degradation types is located and positioned compared to the three obtained RUL histograms. The results presented in Figure 7 demonstrates the efficiency of the prognostic. The sensitivity of the histograms of RUL to the change of the inspection time is evaluated by changing the values of $t_{\text {insp }}$ with $t_{i n s p 1}<t_{i n s p 2}<t_{i n s p 3}$. For a different type of degradation, the inspection time $t_{\text {ins } p}$ will change and the impact of its values is captured in Figure 7 . The real crossing time of the degradation trajectory $R_{c t}$ is presented compared to the histograms of the RUL and also to the confidence intervals of [ $5 \%$ percentile, $95 \%$ percentile]. For slow degradation, the results are presented in the first row of the Figure 7, the real crossing time of the slow degradation path is captured and compared to the three obtained histograms of RUL and to the confidence intervals. As shown for the three histograms, the $R_{c t}$ is well located and conveys an idea about the efficiency of the prognostic. The same work was reproduced for the moderate and fast degradation. Results presented in the second and third rows of Figure 7 prove that for the two types of degradation, the failure of the system can be predicted before it occurs. The $R_{c t}$ of the two degradation paths are well situated compared to the histograms of RUL leading to conclude that the VG proposes an efficient prognostic. The KNN method was used to classify the degradation depending on its speed and for each cluster the prognostic was evaluated. Another classification method is proposed and will be applied on simulated data.

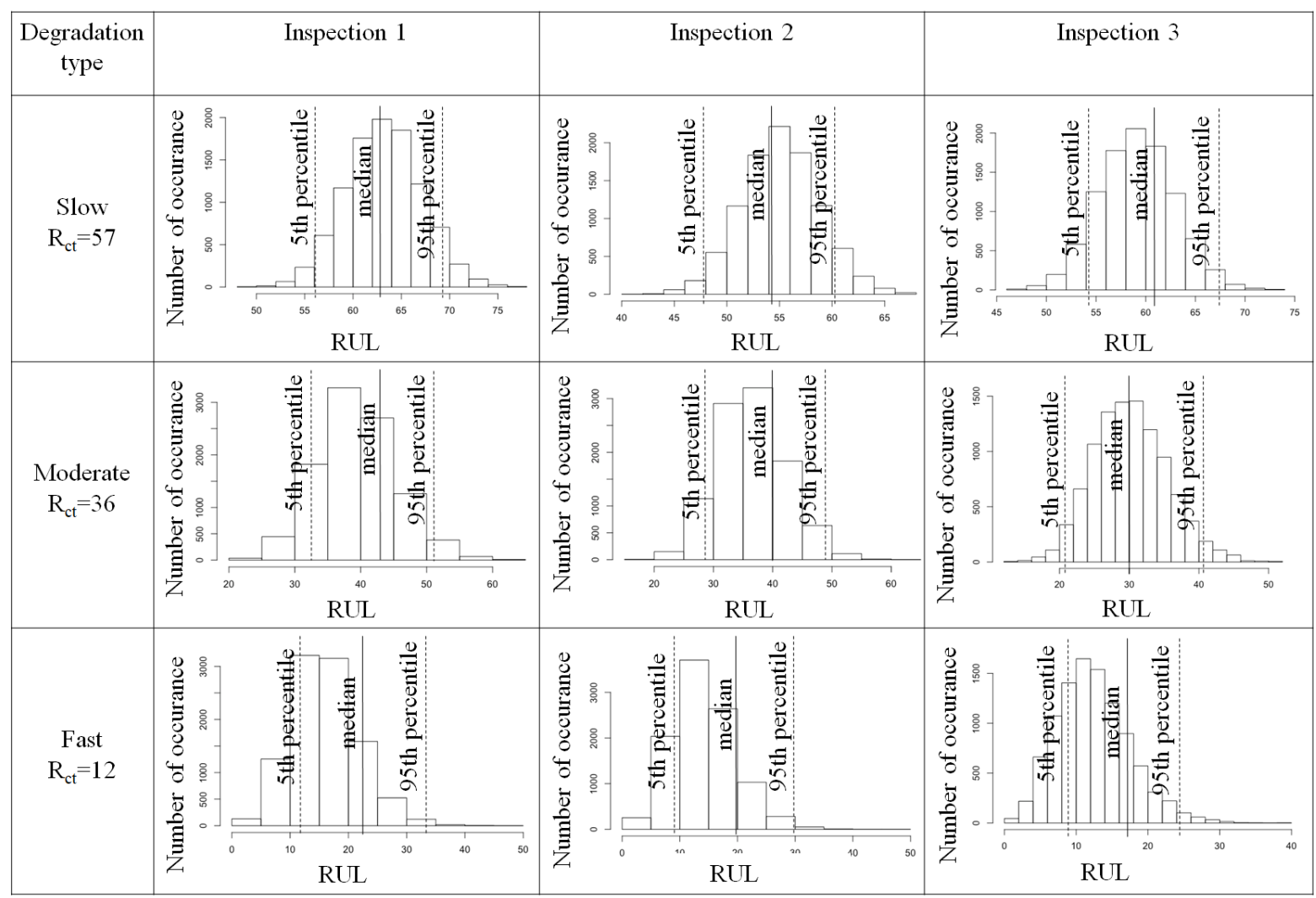

Figure 7. Position of the real crossing time $R_{c t}$ compared to the $5 \%$ and $95 \%$ percentiles of the RUL histograms for simulated data with the KNN algorithm at different inspection times: Slow, moderate, and fast.

\subsubsection{Neural Network Algorithms}

In this study, the neural network algorithm is used to ensure the classification of different degradation paths. The real data base describing the degradation of the centrifugal pump is examined and applied to be classified using the neural network algorithm. As explained before, the neural network algorithm consists of three major layers: First, hidden, and the output layers (Figure 8). The first layer will be assigned to take inputs based on the 
real existing data that will be dispatched to the hidden layers. Later the hidden layers will be replicated using back propagation to get three different clusters describing three types of degradations: Slow, moderate, and fast by optimising the weights of the input variables. The hidden layers are mainly used to improve the predictive power of the neural network model. The output layers are used to output the prediction results based on the data from the input and hidden layers (Figure 8). The architecture, the number of layers, and the number of neurons per layer are considered as hyperparameters of the NN algorithms and different combinations will be tested for obtaining an optimised result. The NN algorithms are applied to detect the type of degradation in this paper. It is crucial in NN algorithm to choose the features that can best present the data and help in detecting the difference between them. The used database is structured based on seven features that best describes the three different degradation types. For a better presentation of the data, the calculation of the mode, mean, skew, variance, kurtosis, as well as the minimum and the maximum of each degradation are presented. The database contains 300 observations with eight variables of which seven present the different features and the last one presents the type of degradation and will be assimilated to the output layer. The classification is based on the seven features introduced as the input layers and evaluated by the algorithm of the hidden layers. The NN algorithm consists of different steps where the first includes the scaling of the original data before applying the algorithm in order to speed up the process and obtain better convergence. The next step is to choose the number of hidden layers, nodes, and other arguments. Then, it is essential to convert a prediction back to the original format leading to creating a confusion matrix and to calculating the classification error.

Using the classification matrix, the error of misclassification can be calculated using the following expression:

$$
\text { error }=1-\frac{\left(d_{11}+d_{22}+\ldots+d_{n n}\right)}{\left(N_{D}\right)}
$$

where $d_{11}, d_{22}, \ldots, d_{n n}$ are the diagonals of the obtained classification matrix and $N_{D}$ is the number of degradation data used for the testing.

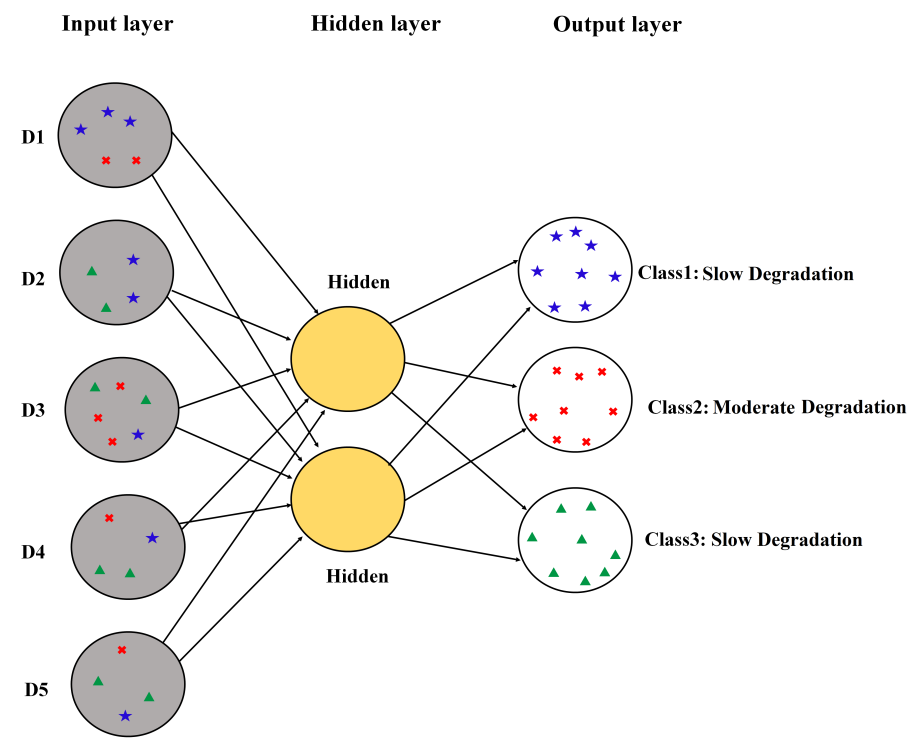

Figure 8. Neural network (NN) methodology.

Figure 9 presents the results of the NN algorithm. The first row presents the first NN architecture. The seven features are introduced as the input layer of the NN algorithm. The hidden layers constitute two layers where the first layer has two nodes and another layer has only one node. The results show that the error of the misclassification calculated using Equation (14) is equal to 0.57. To improve the performance of the algorithm, the NN 
architecture changed to only one hidden layer with four nodes. The second row of the Figure 9 presents the results of the calculation of the NN algorithm, the input nodes will communicate with the four nodes of the hidden layers to ensure the classification. The error of the misclassification of the algorithm is calculated and improved from 0.57 for the previous architecture to 0.45 for the proposed one. The third row of Figure 9 shows the results of calculation of the error for the third architecture. The input nodes will communicate with the hidden layers constituted of two layers: The first with four nodes and the second with two nodes. The error of misclassification is calculated and equal to 0.06 . The best performance of the $\mathrm{NN}$ algorithm was presented by the latest architecture.

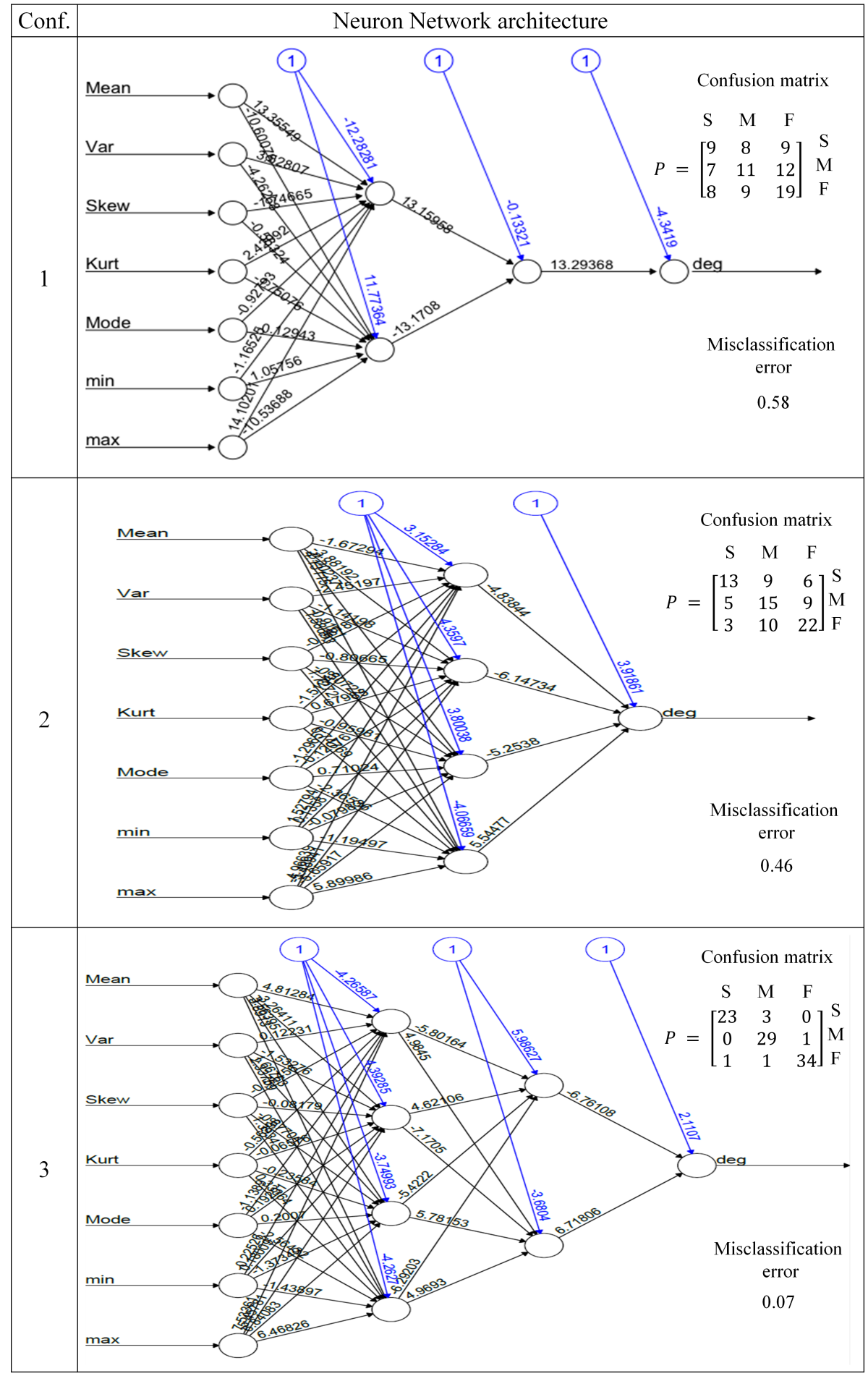

Figure 9. NN classification results for simulated data ( $\mathrm{S}-$ Slow, $\mathrm{M}-$ Moderate, and F-Fast). 
To ensure classification, both the KNN algorithm and NN algorithm were used. Based on the obtained results, the $\mathrm{NN}$ algorithm provided better prediction results equal to $90.4 \%$ compared to the KNN algorithm equal to $90.13 \%$. In most of the cases, the NN algorithm is able to provide better prediction results because of its ability to work with the sized database and big number of features, unlike the KNN algorithm which can prove to have some difficulties.

The efficiency of the classification method is proven, and it is essential to evaluate the quality of the prognostic. The histograms of RUL are evaluated for different VG parameters. Three combinations of VG parameters are proposed and will be used to model the three types of degradation. At $t=0, \mathrm{~N}=10,000$ degradation trajectories are generated and a predefined threshold is settled in order to define the different histograms of RUL. The idea is to assess the evolution of the histograms of RUL at inspection times. First step is to choose the degradation trajectory and to simulate $\mathrm{N}=10,000$ degradation trajectories, once they cross the predefined threshold the first RUL histogram is obtained. At $t=t_{\text {insp }}$, the parameters of the chosen path are estimated and will be used to generate $N=10,000$ trajectories and to obtain the new RUL. The real crossing time of the chosen data will be compared to the two obtained histograms and will provide on the quality of the prognostic. Figure 10 presents the obtained histograms of the RUL for the different degradation paths.

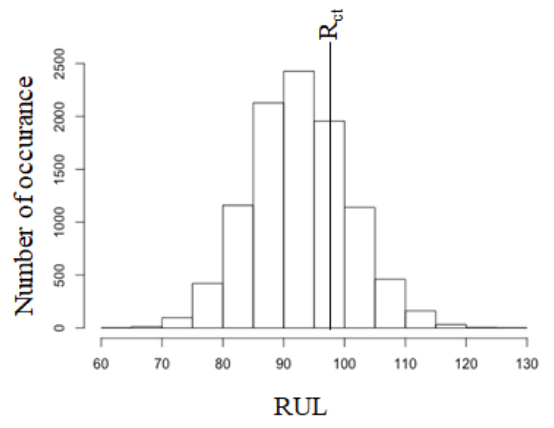

(a)

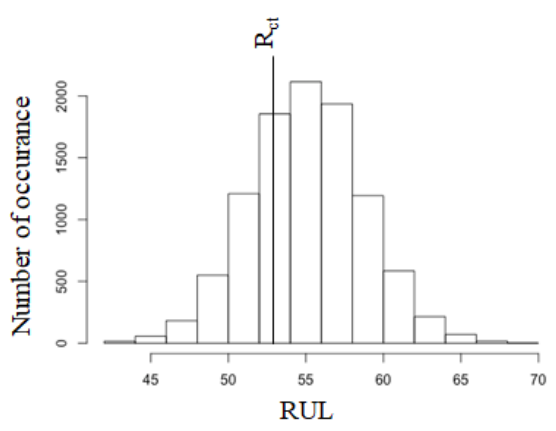

(b)

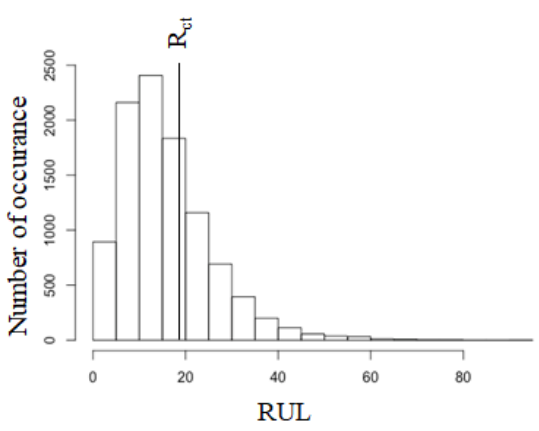

(c)

Figure 10. Position of the real crossing time $R_{c t}$ compared to the histograms of RUL for simulated data with NN algorithm: (a) Slow, (b) moderate, and (c) fast.

For the different degradation types, the real crossing time of the chosen degradation trajectory presented in Figure 10 by $R_{c t}$ is captured and located comparing to the histogram of the RUL. The position of the real crossing time to the histograms of RUL indicates that the VG process can provide a good prognostic. The impact of the change of the inspection time $t_{i n s p}$ on the histograms of RUL is studied and the results are presented in Figure 11. The first row presents the position of the real crossing time $R_{c t}$ of a slow degradation compared to the three histograms of the RUL obtained at three different inspection times. The $R_{c t}$ will also be compared to the confidence intervals [ $\%$ percentile, $95 \%$ percentile]. According to the results, the $R_{c t}$ is positioned in the confidence intervals [5\% percentile, $95 \%$ percentile] of the three histograms obtained at different inspection times. It is possible to predict the failure of the system before it occurs based on the prognostic proposed by VG. Same results were observed for the two other degradation types, the results in Figure 11, shows that the $R_{c t}$ always has a good position compared to the different histograms, conveying the efficiency of the prognostic.

The efficiency of the VG process and the proposed methods were tested using simulated data. It is important to apply the proposed methods on real data. 


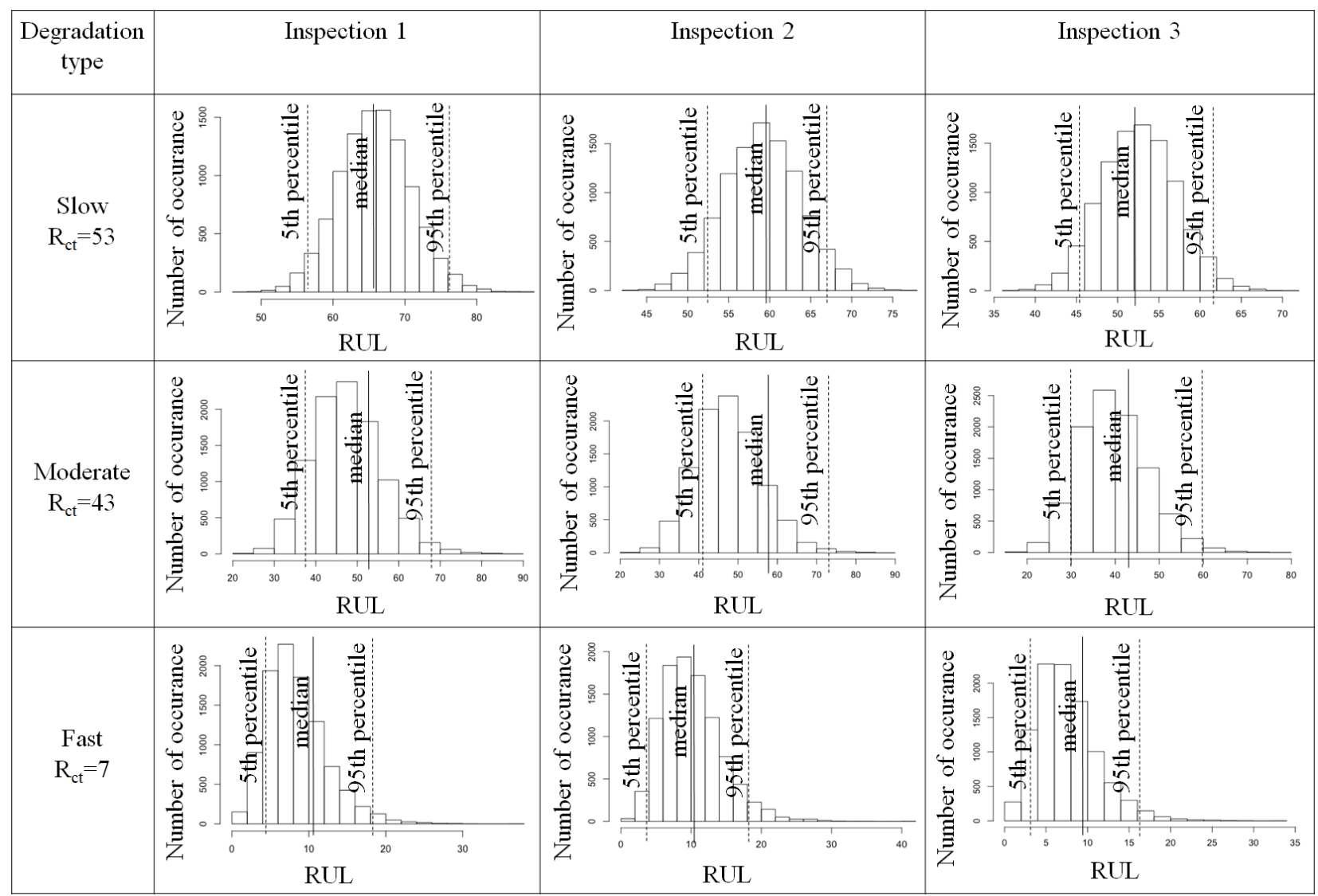

Figure 11. Position of the real crossing time $R_{c t}$ compared to the $5 \%$ and $95 \%$ percentiles of the RUL histograms for simulated data with NN algorithm at different inspection time: Slow, moderate, and fast.

\subsection{Classification Methods for Real Data}

\subsubsection{K-Nearest-Neighbour Algorithms}

In this section the real data will be used to evaluate the performance of the VG process and the proposed classification methods. Based on the real data of seven features are extracted and presented in Table 2. The extracted features contain the VGmod, the VGmean, the VGskew, the VGKurtosis, the VarVG, the minVG, and the maxVG and a target as a type of degradation. The type of degradation is determined based on the values of the other features and will be used to determine the optimal number $K$.

In the case of a small number of neighbours, the noise will have a higher influence on the result, and a large number of neighbours make it computationally expensive. Research works have also shown that a small number of neighbours provide more flexible fittings which will have low bias, but a high variance and a large number of neighbours will have a smoother decision boundary which means a lower variance but higher bias. In this case study, the number $K$ is set to be equal to 4 , the choice was based on different tries and for $K=4$, the results were satisfactory.

Table 3 resumes the results of the KNN prediction accuracy for real data. When the number of neighbours in the model is increased, the accuracy increases till some level. For $K=1$, the KNN prediction accuracy is equal to $66.82 \%$. When the number of $K$ is increased from $K=2$ to $K=4$, the prediction accuracy also increases from $77.92 \%$ to $85.51 \%$. After reaching the value of $K=4$, the increase of its value does not imply an increase in the prediction accuracy. When the number of neighbours is increased to be $K=6, K=8$, and $K=10$, the model did not show better results. This can lead us to conclude that it is not necessary for each case that an increase in many neighbours increases accuracy. It is important to keep the best prediction results obtained for $K=4$ since any misclassification error can induce serious problems in estimating the system's lifetime prognostic. 
Table 2. Features of real data for 16 observations.

\begin{tabular}{|c|c|c|c|c|c|c|c|c|c|c|c|}
\hline \multicolumn{4}{|c|}{ VG Parameters } & \multirow{2}{*}{$\begin{array}{l}\text { Deg. } \\
\text { Type }\end{array}$} & \multirow{2}{*}{$\begin{array}{l}\text { VG } \\
\text { Min }\end{array}$} & \multirow{2}{*}{$\begin{array}{c}\text { VG } \\
\text { Max }\end{array}$} & \multirow{2}{*}{$\begin{array}{l}\text { VG } \\
\text { Mod }\end{array}$} & \multirow{2}{*}{$\begin{array}{c}\text { VG } \\
\text { Mean }\end{array}$} & \multirow{2}{*}{$\begin{array}{c}\text { VG } \\
\text { Skew }\end{array}$} & \multirow{2}{*}{$\begin{array}{c}\text { VG } \\
\text { Kurt }\end{array}$} & \multirow{2}{*}{$\begin{array}{l}\text { VG } \\
\text { Var }\end{array}$} \\
\hline$\sigma$ & $\theta$ & $v$ & $\mu$ & & & & & & & & \\
\hline 0.45 & 0.30 & 0.25 & 0.25 & $S$ & -0.853 & 2.618 & 0.442 & 0.569 & 0.458 & 3.892 & 0.225 \\
\hline 3.5 & 2.5 & 1 & 0.75 & M & -5.249 & 37.592 & 1.75 & 4.250 & 2.507 & 13.530 & 24.75 \\
\hline 0.35 & 0.5 & 0.40 & 0.40 & $S$ & -0.514 & 2.867 & 0.512 & 0.629 & 0.737 & 4.572 & 0.147 \\
\hline 0.25 & 0.75 & 0.25 & 0.15 & $S$ & -0.282 & 1.510 & 0.246 & 0.301 & 0.505 & 3.923 & 0.045 \\
\hline 10 & 9 & 1 & 2 & $\mathrm{~F}$ & -25.036 & 125.741 & 10 & 13.853 & 3.809 & 28.370 & 180 \\
\hline 1 & 1.5 & 0.75 & 1.5 & $S$ & 0.263 & 10.997 & 1.727 & 2.967 & 1.627 & 7.188 & 2.687 \\
\hline 3 & 2 & 2 & 1.5 & M & -7.425 & 25.538 & 1.5 & 3.246 & 2.453 & 13.318 & 17 \\
\hline 10 & 8 & 1 & 2 & $\mathrm{~F}$ & -14.722 & 151.918 & 8 & 14.993 & 4.314 & 31.739 & 315 \\
\hline 5 & 1.5 & 0.5 & 1 & M & -6.583 & 32.533 & 2 & 3.484 & 2.915 & 18.061 & 15.75 \\
\hline 4.75 & 3 & 0.75 & 1 & M & -1.151 & 42.380 & 2 & 5.003 & 2.790 & 14.801 & 22 \\
\hline 7 & 6 & 1 & 2 & $\mathrm{~F}$ & -6.667 & 153.298 & 7 & 11.956 & 4.190 & 30.835 & 129 \\
\hline 3.25 & 2.5 & 1 & 1 & M & -2.945 & 39.808 & 3 & 5.341 & 3.309 & 20.053 & 27.75 \\
\hline 10 & 8 & 1.5 & 2 & $\mathrm{~F}$ & -41.436 & 141.537 & 4 & 8.502 & 4.074 & 30.037 & 225 \\
\hline 0.5 & 0.5 & 0.5 & 0.5 & S & -0.356 & 4.473 & 0.680 & 1.026 & 1.088 & 5.333 & 0.375 \\
\hline 9 & 6 & 1 & 2 & F & -30.599 & 224.604 & 6 & 9.886 & 4.433 & 35.230 & 177 \\
\hline
\end{tabular}

$\sigma, \boldsymbol{\theta}, \boldsymbol{v}$, and $\boldsymbol{\mu}$ are the variance gamma parameters. Deg. type is the degradation type (S: Slow, M: Moderate, and F: Fast). VG Min, VG Max, VG mod, VG mean, VG Skew, VG Kurt, and VG Var are the features.

Table 3. The KNN prediction accuracy for real data.

\begin{tabular}{lcccccc}
\hline $\boldsymbol{K}$ & $\mathbf{1}$ & $\mathbf{2}$ & $\mathbf{4}$ & $\mathbf{6}$ & $\mathbf{8}$ & $\mathbf{1 0}$ \\
\hline Accuracy & 0.6682 & 0.7792 & 0.8551 & 0.7317 & 0.7529 & 0.7261 \\
Classification rate & $66.82 \%$ & $77.92 \%$ & $85.51 \%$ & $73.17 \%$ & $75.29 \%$ & $72.61 \%$ \\
\hline
\end{tabular}

Once the three types of degradation are identified using the KNN algorithm, the rest of the work consists of proposing an efficient prognostic. The first step is to estimate the parameters of the degradation paths. As explained in different studies, the calculation of the maximum likelihood estimation (MLE) of the variance gamma process presented in Equation (6) is quite challenging because of the presence of the Bessel function of the third kind. In that context, the estimation of the VG parameters will be ensured using two R methods named 'ghyp' and 'variance gamma'. The efficiency of the estimation is evaluated using the calculation of the RMSE for both methods. The idea is to choose three degradation paths each one presenting a type of degradation (slow, moderate, and fast) and to calculate the RMSE of their four parameters. RMSE1 presents the results of the calculation of the RMSE provided using the 'ghyp' method, whereas RMSE2 presents those obtained using the 'variance gamma' method. The results of the RMSE calculation for the three degradation paths are presented in Table 4. For the slow degradation path, the results show that the 'ghyp' method provides better results by giving a smaller error estimation compared to the 'variance gamma' $\mathrm{R}$ method. The same results were obtained for the two degradation paths, presenting moderate and fast degradation types. Based on the results of RMSE calculation presented in Table 4 one can conclude that the 'ghyp' method is the best estimation method that can be used since it provides the best estimation results. For the new degradation path, the classification method will be applied, and its unknown parameters will be estimated. Later a detailed study of its first hitting time (FT) distribution is examined and a good prognostic is proposed. 
Table 4. RMSE for different type of trajectories.

\begin{tabular}{|c|c|c|c|c|}
\hline & $\sigma_{R M S E}$ & $\theta_{\text {RMSE }}$ & $v_{R M S E}$ & $\mu_{R M S E}$ \\
\hline \multicolumn{5}{|c|}{$V G_{\text {slow }}(\sigma=0.45, \theta=0.30, v=0.25, \mu=0.25)$} \\
\hline RMSE1 & 0.00058383 & $2.555940 \times 10^{-7}$ & 0.00009207 & 0.00012196 \\
\hline RMSE2 & 0.00080584 & $2.516683 \times 10^{-5}$ & 0.00081257 & 0.0007324230 \\
\hline \multicolumn{5}{|c|}{$V G_{\text {moderate }}(\sigma=3.5, \theta=2.5, v=1, \mu=0.5)$} \\
\hline RMSE1 & $4.063446 \times 10^{-3}$ & 0.00293710 & 0.02806451 & 0.001025312 \\
\hline RMSE2 & $3.870385 \times 10^{-5}$ & 0.09642984 & 0.03979212 & 0.005082687 \\
\hline \multicolumn{5}{|c|}{$V G_{\text {fast }}(\sigma=10, \theta=4, v=1.5, \mu=0.75)$} \\
\hline RMSE1 & 0.001231534 & $2.9477285 \times 10^{-4}$ & 0.001239473 & 0.000463153 \\
\hline RMSE2 & 0.003269308 & $2.936155 \times 10^{-5}$ & 0.001256060 & 0.0006779552 \\
\hline
\end{tabular}

The estimation of the parameters is essential to be considered. The estimated parameters of one chosen degradation path will be used to generate a number $\mathrm{N}=10,000$ of VG degradation paths. When the failure threshold is settled and the degradation paths cross the predefined threshold the time of the first passage time FT will be captured and used to obtain the histogram of the FT as shown in Figure 12.

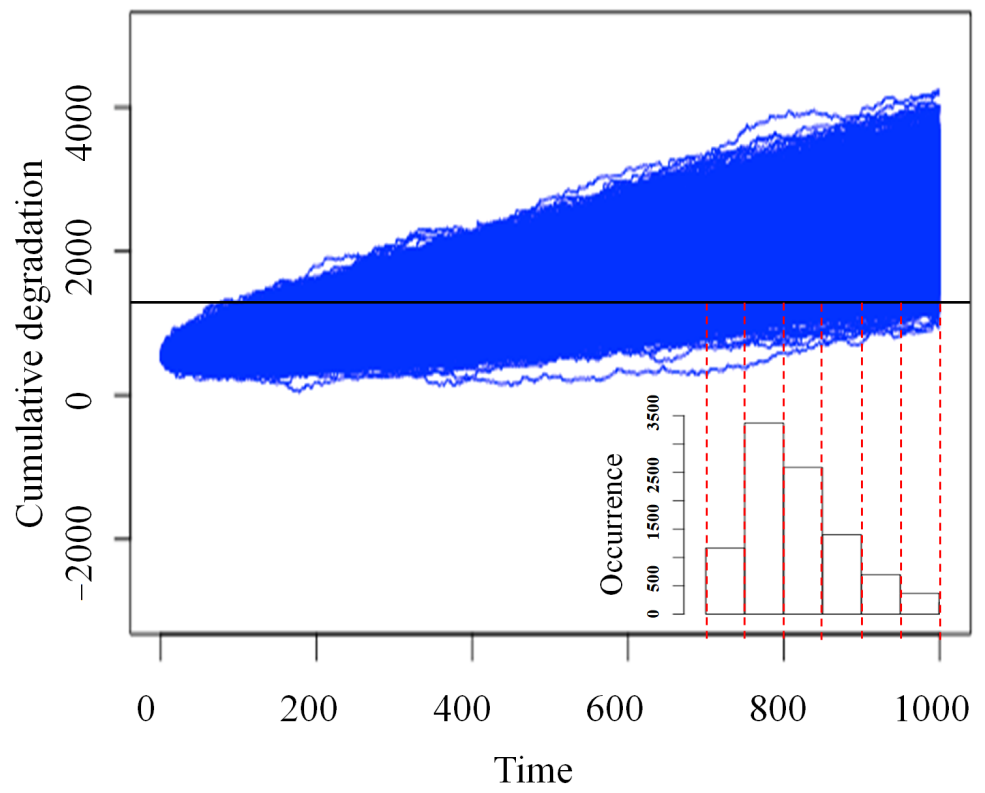

Figure 12. Histogram of RUL for real data.

The same concept used to examine the histogram of the FT will be adopted to evaluate the quality of the prognostic for the VG for the different degradation behaviours. A conclusion that can be drawn from Figure 13 is that for some elements defining a strong degradation, the first crossing time defining the histogram of RUL arrives very quickly implying that the system reaches its failure threshold faster than other types of degradation.

This conclusion can be more justified in Figure 13 where it resumes the impact of the covariates on the histogram of the RUL. The histogram of RUL of the fast degradation reaches the failure threshold faster than any other type of degradation. The time of crossing the failure threshold of the fast degradation occurs before $40 \mathrm{~h}$, whereas for the moderate degradation it happens between 20 and $80 \mathrm{~h}$. Slow degradation data recorded the latest time to achieve the failure threshold. They crossed the failure threshold over the period between 100 and $160 \mathrm{~h}$. Another interpretation can be conducted, the real crossing time $R_{c t}$ of the chosen degradation trajectory presenting the three degradations is compared to the 
histogram of the RUL. The position of the $R_{c t}$ indicates the efficiency of the prognostic. It is important to evaluate the prognostic results when the values of the $t_{\text {insp }}$ change. The first row of Figure 14 presents the results of the comparison of the position of the $R_{c t}$ to the three histograms of RUL. The $R_{c t}$ has a good location in the confidence intervals [5\% percentile, $95 \%$ percentile] of the three histograms allowing a conclusion that the failure of the system can be predicted. The same results were obtained for the two other degradation types, where at the different inspection time $t_{\text {ins }}$ the real crossing time $R_{c t}$ is located in the confidence intervals of the histograms of RUL. Based on the obtained results, the failure of the system modeled using the VG process can be predicted.

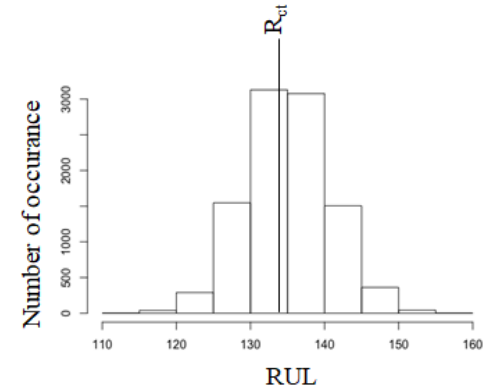

(a)

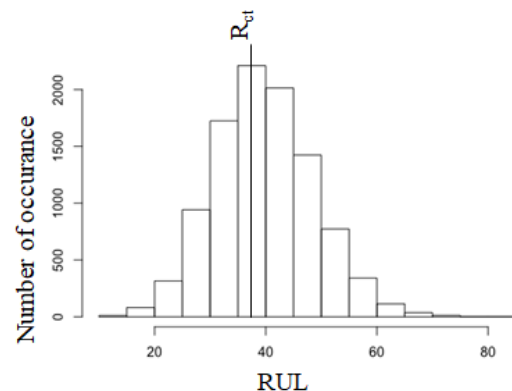

(b)

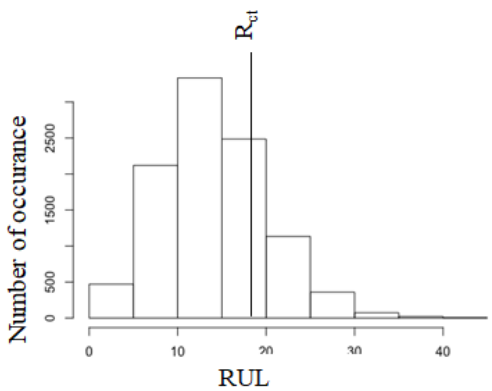

(c)

Figure 13. Position of the real crossing time $R_{c t}$ compared to the histograms of RUL for real data with the KNN algorithm: (a) Slow, (b) moderate, and (c) fast.

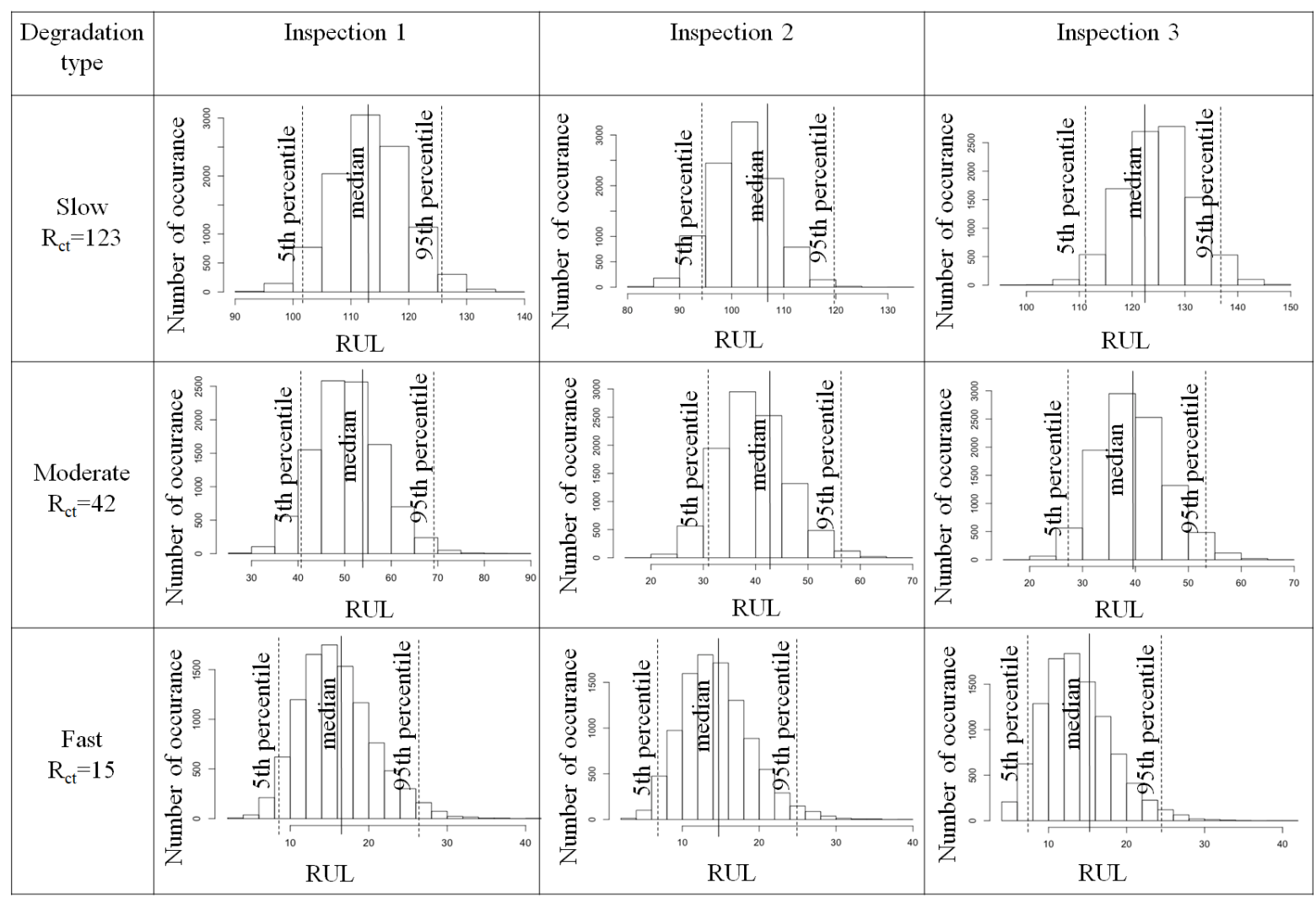

Figure 14. Position of the real crossing time $R_{c t}$ compared to the $5 \%$ and $95 \%$ percentiles of the RUL histograms for real data with the KNN algorithm at different inspection times: Slow, moderate, and fast. 


\subsubsection{Neural Network Algorithms}

As explained, the NN algorithm is applied to detect the type of centrifugal pump degradation and the results are presented in Figure 15. The full data will be divided into two parts: Training data and testing data. In the training phase, 208 data are used to ensure the training of the algorithm while 92 will ensure the testing task. Based on the first row in Figure 15, the seven features were used as an input layer of the architecture, then the hidden layers were only presented by one layer with two nodes which communicate with the output layer used to illustrate the degradation type. The aim of the algorithm is to predict the future classification, so by using the testing data the prediction is calculated. The results of the prediction calculation are applied and used to create the confusion matrix. The results presented in the first row of Figure 15 shows that the algorithm was able to predict that 10 slow degradation were predicted to be slow same 16 moderate degradation and 20 fast degradation were successfully predicted. The algorithm show that eight moderate and four fast degradation types were predicted as slow degradation which constitute a misclassification.

When the error equation is calculated for the first row of Figure 15 the algorithm shows that the error is equal to 0.5 . To improve the accuracy of the algorithm, the same work will be reproduced with a superior number of nodes. The number of nodes of the hidden layer passed from 2 to 5 and the results are presented in the second row of the Figure 15. The input will communicate with a hidden layer of five nodes, the results improved and the error of the misclassification passes from 0.5 to 0.34 . The confusion matrix also shows that for the same data the $\mathrm{NN}$ algorithm was able to detect more correctly the type of degradation. For this configuration the number of real slow, moderate, and fast degradations predicted increased which minimise the number of data misclassified. The third row of Figure 15 resumes a new architecture of the NN algorithm. In this part, two hidden layers are used with five nodes for the first one and two nodes for the second one. The confusion matrix explains that the proposed NN algorithm is able to successfully predict the degradation type. The misclassification error is equal to 0.12 which can be a satisfactory result for the classification algorithm. The aim is to test the NN algorithm to reduce the misclassification error. Another architecture was tested where the number of nodes of the second layer will increase. For NN with two hidden layers, one contains five nodes and the second with three nodes. This NN algorithm was not able to converge which lead to accepting the results obtained by the previous architecture of the $\mathrm{NN}$ algorithm. The best architecture of the NN algorithm was chosen, and gave a prediction accuracy equal to $88 \%$. Both KNN and NN methods were applied as classification methods for the system degradation paths and it is noticeable that the $\mathrm{NN}$ algorithm provided better results in comparison to the KNN algorithm. The prediction accuracy of the NN algorithm for real data is equal to $88 \%$ whereas the prediction accuracy of the $\mathrm{KNN}$ algorithm is equal to $85.51 \%$. This result can be explained based on the fact that the NN algorithm is more efficient than the KNN algorithm when the number of data is important. One of the shortcomings of the KNN algorithm is that it is slow and does not work as expected when working with a big number of features or parameters.

Once the classification results are accepted, the evaluation of the prognostic will be assessed. For the different degradation paths classified using the NN algorithm, three chosen degradation trajectories will be used to evaluate the prognostic. At $t=0$, the VG parameters are used to generate $\mathrm{N}=10,000$ degradation trajectories and once they reach the predefined threshold the histograms of RUL are captured. The same work will be reproduced at $t=t_{\text {insp }}$ and the position of the real crossing time of the initial degradation path will be compared to the two histograms. Figure 16 provides the histograms of the RUL and gives an idea about the quality of the prognostic. Figure 16 resumes the position of the real crossing time $R_{c t}$ compared to the obtained histograms of the RUL. For the different histograms the $R_{c t}$ is positioned almost near their different medians which imply the efficiency of the prognostic. 


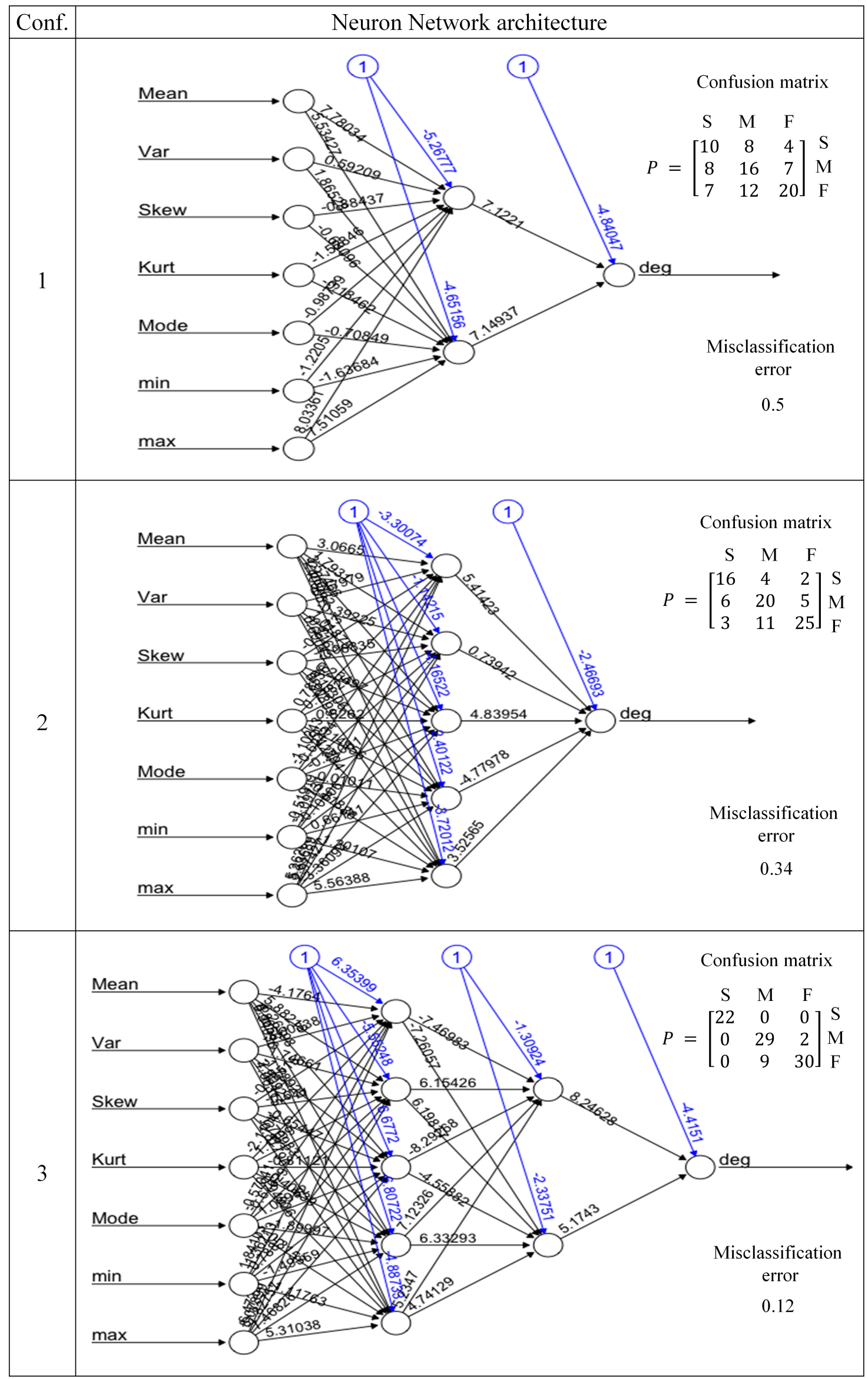

Figure 15. NN prediction results for real data: (S-Slow, M-Moderate, and F-Fast). 
To investigate more about the quality of the prognostic, the histograms of RUL will be generated at different inspection times $t_{i n s p}$ and the real crossing time $R_{c t}$ will be compared to them. The histograms of RUL of three degradation types are evaluated, and the results are presented in Figure 17. For the slow degradation, the results presented in the first row show that the real crossing time $R_{c t}$ has a good position compared to the three confidence intervals [ $5 \%$ percentile, $95 \%$ percentile] of the different histograms of RUL. The same results were observed for the moderate degradation where the $R_{c t}$ is located in the confidence intervals and encountered by the histograms of RUL. Similar results were observed for the fast degradation, which leads to the conclusion that the failure of the system can be observed and detected before it happens. Thus the prognostic provided using the VG process can be considered as efficient since it detects the failure before it occurs.

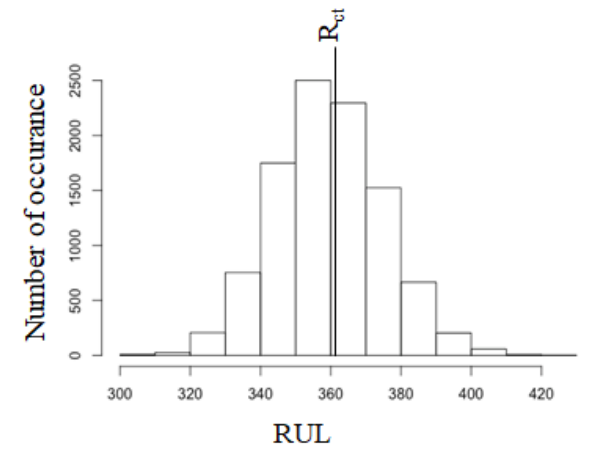

(a)

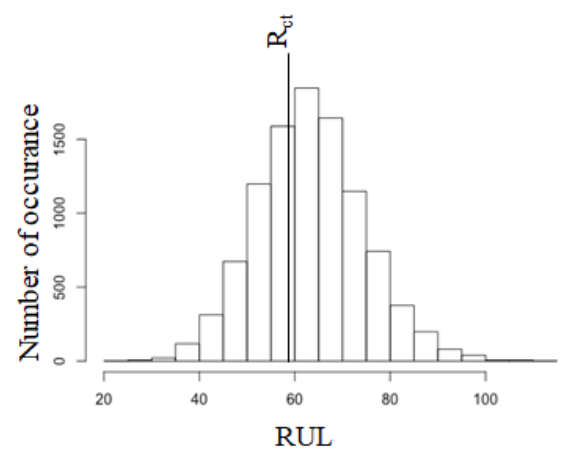

(b)

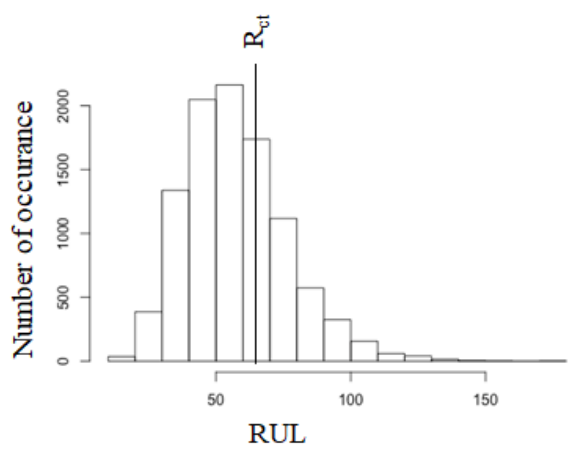

(c)

Figure 16. Position of the real crossing time $R_{c t}$ compared to the histograms of RUL for real data with the NN algorithm: (a) Slow, (b) moderate, and (c) fast.

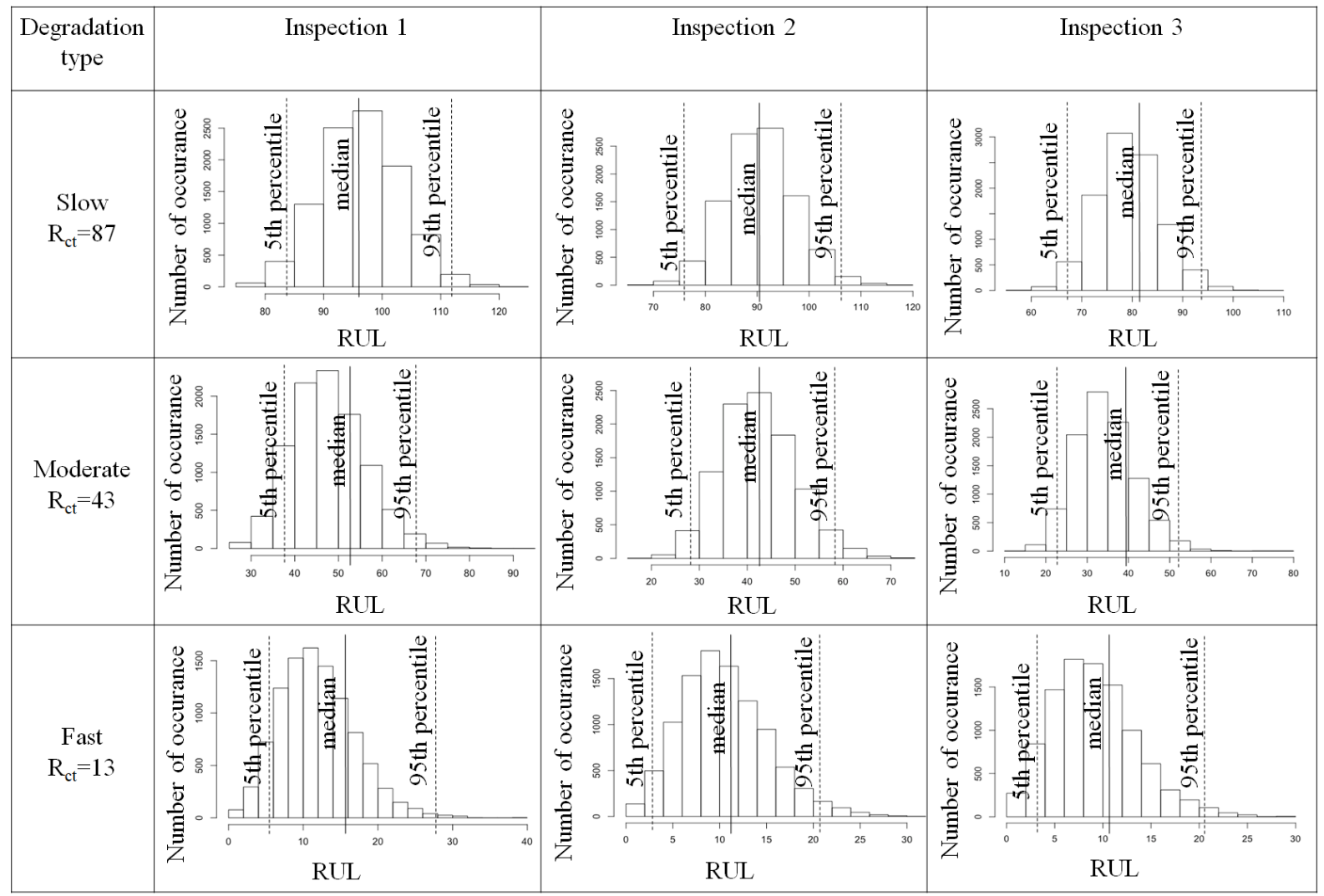

Figure 17. Position of the real crossing time $R_{c t}$ compared to the $5 \%$ and $95 \%$ percentiles of the RUL histograms for real data with the NN algorithm at different inspection times: Slow, moderate, and fast. 


\section{Conclusions}

In this study, the VG process is proposed to model a mechanical system degradation. To investigate its feasibility, real degradation data of a centrifugal pump were used. Initially, the VG process, its definition, and properties were presented. The choice of the degradation model was based on an understanding of the operating mode of the system in degradation. Several researchers investigated the impact of the environment of the system degradation and encouraged the incorporation of the environmental effects in degradation modelling. The effect of the environment such as temperature, humidity, and pressure was described by covariates and could be modelled via different models. In the paper the behaviour of the centrifugal pump operating in a dynamic environment was analysed. The presence of covariates was presented using a Markov Chain and both variance Gamma process and Markov chain was used as the degradation model. The covariates were used to describe the speed of the degradation: Slow, moderate, and fast. First, the unknown parameters of the model were estimated. The mixture distribution method was applied, and the maximum likelihood estimation examined. The estimation was a challenging task because of the presence of the Bessel function of the third kind in the expression of the MLE. To overcome the problem of estimating the supervised classification method, the KNN was proposed. The aim was to classify the degradation data on three clusters presenting the three different degradation speeds. After the classification, the estimation of the unknown parameters of the model and the FT distribution was examined. Moreover, the evolution of the prognostic depending on the degradation speed was also presented. Finally, the KNN and NN algorithms were also tested to classify the new degradation paths based on its type of degradation. In further study, it will be judicious to study more the efficiency of the VG process as a mathematical model while testing an efficient maintenance policy. It will also be important to propose different maintenance policies for the three different clusters and to procure the possibility for maintenance practitioners using KNN and NN algorithms of identifying the type of degradation and the relative maintenance actions.

Author Contributions: Conceptualisation, M.B.S. and M.F.; software, M.B.S.; writing-original draft preparation, M.B.S.; writing-review and editing, M.F. and E.D. All authors have read and agreed to the published version of the manuscript.

Funding: This research was supported by the European Regional Development Fund (FEDER) and the Departmental Council of Aube.

Institutional Review Board Statement: Not applicable.

Informed Consent Statement: Not applicable.

Data Availability Statement: Not applicable.

Acknowledgments: The authors would like to thank the anonymous reviewers for their useful comments that have improved the final version of this manuscript.

Conflicts of Interest: The authors declare no conflict of interest.

\section{References}

1. Bogdanoff, J.L.; Kozin, F. Probabilistic Models of Cumulative Damage; Wiley-Interscience: New York, NY, USA, 1985; 350p.

2. Van Noortwijk, J.M. A survey of the application of gamma processes in maintenance. Reliab. Eng. Syst. Saf. 2009, 94, 2-21. [CrossRef]

3. Frangopol, D.M.; Kallen, M.J.; Noortwijk, J.M.V. Probabilistic models for life-cycle performance of deteriorating structures: Review and future directions. Prog. Struct. Eng. Mater. 2004, 6, 197-212. [CrossRef]

4. Abdel-Hameed, M. Degradation processes: An overview. In Advances in Degradation Modeling; Springer: Berlin/Heidelberg, Germany, 2010; pp. 17-25.

5. Madan, D.B.; Seneta, E. The variance gamma (VG) model for share market returns. J. Bus. 1990, 63, 511-524. [CrossRef]

6. Madan, D.B.; Milne, F. Option pricing with vg martingale components 1. Math. Financ. 1991, 1, 39-55. [CrossRef]

7. Madan, D.B.; Carr, P.P.; Chang, E.C. The variance gamma process and option pricing. Rev. Financ. 1998, 2, 79-105. [CrossRef]

8. Fiorani, F. The Variance-Gamma process for option pricing. J. Comput. Financ. 1999, 2, 61-73. [CrossRef]

9. Fiorani, F. Option Pricing Under the Variance Gamma Process. 2004. Available online: https://ssrn.com/abstract=1411741 (accessed on 23 November 2020). 
10. Yoo, E. Variance Gamma Pricing of American Futures Options; Florida State University: Tallahassee, FL, USA, 2008.

11. Marfè, R. A generalized variance gamma process for financial applications. Quant. Financ. 2012, 12, 75-87. [CrossRef]

12. Rathgeber, A.W.; Stadler, J.; Stöckl, S. Modeling share returns-an empirical study on the Variance Gamma model. J. Econ. Financ. 2016, 40, 653-682. [CrossRef]

13. Nitithumbundit, T.; Chan, J.S. ECM Algorithm for Auto-Regressive Multivariate Skewed Variance Gamma Model with Unbounded Density. Methodol. Comput. Appl. Probab. 2019, 22, 1169-1191. [CrossRef]

14. Whitley, A. Pricing of European, Bermudan and American Options under the Exponential Variance Gamma Process. 2009. Available online: https:/ / ora.ox.ac.uk/objects/uuid:7e32bbe5-eb07-442b-bbc4-e1d496ac8aab (accessed on 23 November 2020).

15. Bollin, B.; Ślepaczuk, R. Variance Gamma Model in Hedging Vanilla and Exotic Options; Technical Report; 2020. Available online: https:/ / www.wne.uw.edu.pl/files/8416/0034/9047/WNE_WP337.pdf (accessed on 23 November 2020).

16. Cox, D.R. Regression models and life-tables. J. R. Stat. Soc. Ser. B 1972, 34, 187-202. [CrossRef]

17. Deloux, E.; Castanier, B.; Bérenguer, C. Predictive maintenance policy for a gradually deteriorating system subject to stress. Reliab. Eng. Syst. Saf. 2009, 94, 418-431. [CrossRef]

18. Zhao, X.; Gaudoin, O.; Doyen, L.; Xie, M. Optimal inspection and replacement policy based on experimental degradation data with covariates. IISE Trans. 2019, 51, 322-336. [CrossRef]

19. Barabadi, A.; Barabady, J.; Markeset, T. Application of reliability models with covariates in spare part prediction and optimizationA case study. Reliab. Eng. Syst. Saf. 2014, 123, 1-7. [CrossRef]

20. Okaro, I.A.; Tao, L. Reliability analysis and optimisation of subsea compression system facing operational covariate stresses. Reliab. Eng. Syst. Saf. 2016, 156, 159-174. [CrossRef]

21. Moniri-Morad, A.; Pourgol-Mohammad, M.; Aghababaei, H.; Sattarvand, J. Reliability-based covariate analysis for complex systems in heterogeneous environment: Case study of mining equipment. Proc. Inst. Mech. Eng. Part O J. Risk Reliab. 2019, 233, 593-604. [CrossRef]

22. Slimacek, V.; Lindqvist, B. Reliability of wind turbines modeled by a Poisson process with covariates, unobserved heterogeneity and seasonality. Wind Energy 2016, 19, 1991-2002. [CrossRef]

23. Duan, F.; Wang, G. Exponential-dispersion degradation process models with random effects and covariates. IEEE Trans. Reliab. 2018, 67, 1128-1142. [CrossRef]

24. Barabadi, A.; Barabady, J.; Markeset, T. Maintainability analysis considering time-dependent and time-independent covariates. Reliab. Eng. Syst. Saf. 2011, 96, 210-217. [CrossRef]

25. Guo, R.; Love, E. Reliability modelling with fuzzy covariates. Int. J. Reliab. Qual. Saf. Eng. 2003, 10, 131-157. [CrossRef]

26. Zhu, W.; Fouladirad, M.; Bérenguer, C. Condition-based maintenance policies for a combined wear and shock deterioration model with covariates. Comput. Ind. Eng. 2015, 85, 268-283. [CrossRef]

27. Zhang, N.; Fouladirad, M.; Barros, A. Reliability-based measures and prognostic analysis of a K-out-of-N system in a random environment. Eur. J. Oper. Res. 2019, 272, 1120-1131. [CrossRef]

28. Lawless, J.; Crowder, M. Covariates and random effects in a gamma process model with application to degradation and failure. Lifetime Data Anal. 2004, 10, 213-227. [CrossRef] [PubMed]

29. Laucelli, D.; Rajani, B.; Kleiner, Y.; Giustolisi, O. Study on relationships between climate-related covariates and pipe bursts using evolutionary-based modelling. J. Hydroinform. 2014, 16, 743-757. [CrossRef]

30. Park, S.; Jun, H.; Agbenowosi, N.; Kim, B.J.; Lim, K. The proportional hazards modeling of water main failure data incorporating the time-dependent effects of covariates. Water Resour. Manag. 2011, 25, 1-19. [CrossRef]

31. Balekelayi, N.; Tesfamariam, S. Statistical inference of sewer pipe deterioration using Bayesian geoadditive regression model. J. Infrastruct. Syst. 2019, 25, 04019021. [CrossRef]

32. Gorjian, N.; Rameezdeen, R.; Gorjian Jolfaei, N.; Chow, C.; Jin, B. Ref: EATJ-D-19-00148-prediction of remaining useful life of naval structures using a covariate-base hazard model. Aust. J. Struct. Eng. 2020, 1-10. [CrossRef]

33. Xu, Z.; Hong, Y.; Jin, R. Nonlinear general path models for degradation data with dynamic covariates. Appl. Stoch. Model. Bus. Ind. 2016, 32, 153-167. [CrossRef]

34. Zhou, B.; Qi, F.; Tao, H. Condition-based maintenance modeling for a two-stage deteriorating system with random changes based on stochastic process. J. Qual. Maint. Eng. 2017, 23. [CrossRef]

35. Liu, B.; Zhao, X.; Liu, G.; Liu, Y. Life cycle cost analysis considering multiple dependent degradation processes and environmental influence. Reliab. Eng. Syst. Saf. 2020, 197, 106784. [CrossRef]

36. Giorgio, M.; Guida, M.; Pulcini, G. A new class of Markovian processes for deteriorating units with state dependent increments and covariates. IEEE Trans. Reliab. 2015, 64, 562-578. [CrossRef]

37. Arabani, M.; Manavizadeh, N.; Balali, S. A Stochastic Model for Indirect Condition Monitoring Using Proportional Covariate Model. Int. J. Eng. Trans. A Basics 2007, 21, 45-56.

38. Mahmoodi, S.; Hamed Ranjkesh, S.; Zhao, Y.Q. Condition-based maintenance policies for a multi-unit deteriorating system subject to shocks in a semi-Markov operating environment. Qual. Eng. 2020, 1-12. [CrossRef]

39. Ahmadzadeh, F.; Ghodrati, B.; Kumar, U. Mean residual life estimation considering operating environment. In Proceedings of the International Conference on Quality, Reliability, Infocom Technology and Industrial Technology Management, Newdelhi, India, 26-28 November 2012. 
40. Shahraki, A.F.; Yadav, O.P. Selective maintenance optimization for multi-state systems operating in dynamic environments. In Proceedings of the 2018 Annual Reliability and Maintainability Symposium (RAMS), IEEE, Reno, NV, USA, 22-25 January 2018; pp. 1-6.

41. Si, W.; Love, E.; Yang, Q. Two-state optimal maintenance planning of repairable systems with covariate effects. Comput. Oper. Res. 2018, 92, 17-25. [CrossRef]

42. Tang, D.; Makis, V.; Jafari, L.; Yu, J. Optimal maintenance policy and residual life estimation for a slowly degrading system subject to condition monitoring. Reliab. Eng. Syst. Saf. 2015, 134, 198-207. [CrossRef]

43. Huynh, K.T.; Barros, A.; Bérenguer, C. Adaptive condition-based maintenance decision framework for deteriorating systems operating under variable environment and uncertain condition monitoring. Proc. Inst. Mech. Eng. Part O J. Risk Reliab. 2012, 226, 602-623. [CrossRef]

44. Wu, S.; Scarf, P. Decline and repair, and covariate effects. Eur. J. Oper. Res. 2015, 244, 219-226. [CrossRef]

45. Hong, Y.; Duan, Y.; Meeker, W.Q.; Stanley, D.L.; Gu, X. Statistical methods for degradation data with dynamic covariates information and an application to outdoor weathering data. Technometrics 2015, 57, 180-193. [CrossRef]

46. Si, W.; Shao, Y.; Wei, W. Accelerated Degradation Testing With Long-Term Memory Effects. IEEE Trans. Reliab. 2020, 69, 1254-1266. [CrossRef]

47. Aalen, O.O.; Borgan, Ø.; Fekjær, H. Covariate adjustment of event histories estimated from Markov chains: The additive approach. Biometrics 2001, 57, 993-1001. [CrossRef]

48. Islam, M.A.; Chowdhury, R.I. A higher order Markov model for analyzing covariate dependence. Appl. Math. Model. 2006, 30, 477-488. [CrossRef]

49. Sirima, P.; Pokorny, P. Hidden Markov models with covariates for analysis of defective industrial machine parts. J. Math. Stat. 2014, 10, 322-330. [CrossRef]

50. Zhao, X.; Fouladirad, M.; Bérenguer, C.; Bordes, L. Condition-based inspection/replacement policies for non-monotone deteriorating systems with environmental covariates. Reliab. Eng. Syst. Saf. 2010, 95, 921-934. [CrossRef]

51. Deloux, E.; Fouladirad, M.; Bérenguer, C. Health-and-usage-based maintenance policies for a partially observable deteriorating system. Proc. Inst. Mech. Eng. Part O J. Risk Reliab. 2016, 230, 120-129. [CrossRef]

52. Cover, T.; Hart, P. Nearest neighbor pattern classification. IEEE Trans. Inf. Theory 1967, 13, 21-27. [CrossRef]

53. Di Lucca, G.A.; Di Penta, M.; Gradara, S. An approach to classify software maintenance requests. In Proceedings of the International Conference on Software Maintenance, Montreal, QC, Canada, 3-6 October 2002; IEEE: New York, NY, USA, 2002; pp. 93-102.

54. Yao, Y.; Tang, X.; Lim, E.P. Localized monitoring of kNN queries in wireless sensor networks. VLDB J. 2009, 18, 99-117. [CrossRef]

55. Wang, H.; Yu, Z.; Guo, L. Real-time Online Fault Diagnosis of Rolling Bearings Based on KNN Algorithm. J. Phys. Conf. Ser. 2020, 1486, 032019. [CrossRef]

56. Lee, J. Measurement of machine performance degradation using a neural network model. Int. J. Model. Simul. 1996, 16, 192-199. [CrossRef]

57. Huang, Y.H. Artificial neural network model of bridge deterioration. J. Perform. Constr. Facil. 2010, 24, 597-602. [CrossRef]

58. Quah, T.S.; Thwin, M.M.T. Application of neural networks for software quality prediction using object-oriented metrics. In Proceedings of the International Conference on Software Maintenance, ICSM 2003, Amsterdam, The Netherlands, 22-26 September 2003; IEEE: New York, NY, USA, 2003; pp. 116-125.

59. Hurd, T.R. Credit risk modeling using time-changed Brownian motion. Int. J. Theor. Appl. Financ. 2009, 12, 1213-1230. [CrossRef]

60. Hurd, T.; Kuznetsov, A. On the first passage time for Brownian motion subordinated by a Lévy process. J. Appl. Probab. 2009, 46, 181-198. [CrossRef]

61. Li, H. First-Passage Time Models with a Stochastic Time Change in Credit Risk. In Theses and Dissertations (Comprehensive); Wilfrid Laurier University: Waterloo, ON, Canada, 2009. 